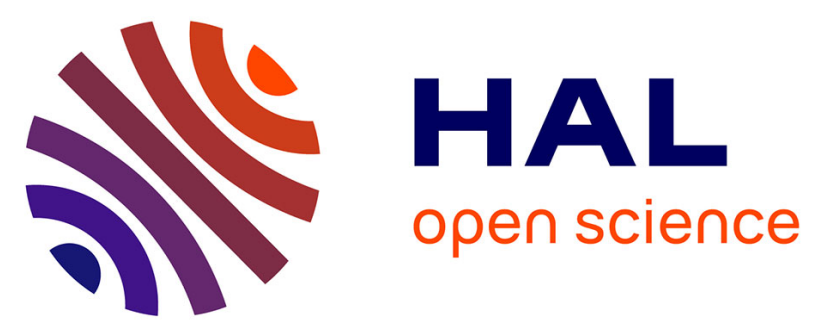

\title{
Linking atomic and mesoscopic scales for the modelling of the transport properties of uranium dioxide under irradiation
}

\author{
M. Bertolus, Michel Freyss, Boris Dorado, Kiet Hoang, Serge Maillard, \\ Richard Skorek, Philippe Garcia, C. Valot, Alain Chartier, Laurent van \\ Brutzel, et al.
}

\section{To cite this version:}

M. Bertolus, Michel Freyss, Boris Dorado, Kiet Hoang, Serge Maillard, et al.. Linking atomic and mesoscopic scales for the modelling of the transport properties of uranium dioxide under irradiation. Journal of Nuclear Materials, 2015, 462, pp.475-495. 10.1016/j.jnucmat.2015.02.026 . cea-02066505

\section{HAL Id: cea-02066505 \\ https://hal-cea.archives-ouvertes.fr/cea-02066505}

Submitted on 13 Mar 2019

HAL is a multi-disciplinary open access archive for the deposit and dissemination of scientific research documents, whether they are published or not. The documents may come from teaching and research institutions in France or abroad, or from public or private research centers.
L'archive ouverte pluridisciplinaire HAL, est destinée au dépôt et à la diffusion de documents scientifiques de niveau recherche, publiés ou non, émanant des établissements d'enseignement et de recherche français ou étrangers, des laboratoires publics ou privés. 


\title{
Linking atomic and mesoscopic scales for the modelling of the transport properties of uranium dioxide under irradiation
}

\author{
Marjorie Bertolus $^{\mathrm{a}, *}$, Michel Freyss ${ }^{\mathrm{a}}$, Boris Dorado ${ }^{\mathrm{a}}$, Guillaume Martin ${ }^{\mathrm{a}}$, Kiet Hoang ${ }^{\mathrm{a}}$, Serge Maillard ${ }^{\mathrm{a}}$, \\ Richard Skorek $^{\mathrm{a}}$, Philippe Garcia ${ }^{\mathrm{a}}$, Carole Valot ${ }^{\mathrm{a}}$, Alain Chartier ${ }^{\mathrm{b}}$, Laurent Van Brutzel ${ }^{\mathrm{b}}$, Paul Fossati ${ }^{\mathrm{b}}$, \\ Robin W. Grimes ${ }^{\text {, }}$, David C. Parfitt ${ }^{\text {, }}$, Clare L. Bishop ${ }^{c}$, Samuel T. Murphy ${ }^{\text {, }}$, Michael J.D. Rushton ${ }^{\text {, }}$, \\ Dragos Staicu $^{d}$, Eugen Yakub ${ }^{d}$, Sergii Nichenko ${ }^{d}$, Matthias Krack ${ }^{\mathrm{e}}$, Fabien Devynck ${ }^{\mathrm{e}}$, \\ Raoul Ngayam-Happy ${ }^{\mathrm{e}}$, Kevin Govers ${ }^{\mathrm{f}}$, Chaitanya S. Deo ${ }^{\mathrm{g}}$, Rakesh K. Behera ${ }^{\mathrm{g}}$
}

${ }^{a} C E A, D E N, D E C / S E S C$, Centre de Cadarache, 13108 Saint-Paul-lez-Durance, France

${ }^{\mathrm{b}}$ CEA, DEN, DPC/SCCME, 91191 Gif-sur-Yvette, France

${ }^{\mathrm{c}}$ Department of Materials, Imperial College London, London SW7 2AZ, United Kingdom

d European Commission, Joint Research Centre, Institute for Transuranium Elements, 76125 Karlsruhe, Germany

e Laboratory for Reactor Physics and Systems Behaviour, Paul Scherrer Institute, CH-5232 Villigen PSI, Switzerland

${ }^{\mathrm{f}}$ Belgian Nuclear Research Center (SCK.CEN), Boeretang 200, B-2400 Mol, Belgium

${ }^{\mathrm{g}}$ Nuclear and Radiological Engineering Program, George W. Woodruff School of Mechanical Engineering, Georgia Institute of Technology, Atlanta, GA 30332, USA

\section{A R T I C L E I N F O}

Article history:

Available online 25 February 2015

\begin{abstract}
A B S T R A C T
This article presents a synthesis of the investigations at the atomic scale of the transport properties of defects and fission gases in uranium dioxide, as well as of the transfer of results from the atomic scale to models at the mesoscopic scale, performed during the F-BRIDGE European project (2008-2012).

We first present the mesoscale models used to investigate uranium oxide fuel under irradiation, and in particular the cluster dynamics and kinetic Monte Carlo methods employed to model the behaviour of defects and fission gases in $\mathrm{UO}_{2}$, as well as the parameters of these models. Second, we describe briefly the atomic scale methods employed, i.e. electronic structure calculations and empirical potential methods. Then, we show the results of the calculation of the data necessary for the mesoscale models using these atomic scale methods. Finally, we summarise the links built between the atomic and mesoscopic scale by listing the data calculated at the atomic scale which are to be used as input in mesoscale modelling.

Despite specific difficulties in the description of fuel materials, the results obtained in F-BRIDGE show that atomic scale modelling methods are now mature enough to obtain precise data to feed higher scale models and help interpret experiments on nuclear fuels. These methods bring valuable insight, in particular the formation, binding and migration energies of point and extended defects, fission product localization, incorporation energies and migration pathways, elementary mechanisms of irradiation induced processes. These studies open the way for the investigation of other significant phenomena involved in fuel behaviour, in particular the thermochemical and thermomechanical properties and their evolution in-pile, complex microstructures, as well as of more complex fuels.
\end{abstract}

(c) 2015 Elsevier B.V. All rights reserved.

\section{Introduction}

The F-BRIDGE project $[1,2]$ was a European initiative aiming at complementing the classical empirical approach to design nuclear fuels by a more physically-based description of fuel and cladding materials. As such, F-BRIDGE was part of the international on-going effort to increase the efficiency in designing innovative

\footnotetext{
* Corresponding author. Tel.: +334422529 33; fax: +33442253285.

E-mail address: marjorie.bertolus@cea.fr (M. Bertolus).
}

fuels both to improve performance and safety of current fuel systems and to design those for tomorrow, next to the Center for Material Science of Nuclear Fuels (CMSNF) [3], the Nuclear Energy Advanced Modelling and Simulation (NEAMS) program [4], the Consortium for Advanced Simulation of Light Water Reactors (CASL) [5] in the US, or the NXO project in Japan [6].

A better knowledge of the elementary phenomena occurring under irradiation is crucial to enable a better prediction, leading itself to a rationalization of the design process and a better selection of promising fuel systems. One important objective of the 
F-BRIDGE European project was to develop a consistent multiscale approach enabling the description of the transport properties in $\mathrm{UO}_{2}$ under irradiation (in particular normal operational conditions) at the mesoscopic level. Various methods from the atomic to the mesoscopic scale, which yield complementary information, were used, in particular electronic structure methods (density functional theory), empirical potentials, kinetic Monte Carlo methods and rate theory models (cluster dynamics). The results of atomistic simulations represented the keystones and guiding principles for the establishment of the mesoscale models, but the analysis of the mesoscale models input parameters also guided the investigations performed at the atomic scale. This multiscale approach was developed jointly with the experimental separate effect studies and characterizations at relevant scales also performed in the F-BRIDGE project $[7,8]$.

The multiscale approach that the F-BRIDGE investigation contributed to develop is shown in Fig. 1. The green rectangle shows the methods used for the investigation of transport properties reported here.

We present here a synthesis of the investigations at the atomic scale of the transport properties of defects and fission gases in uranium dioxide, as well as of the transfer of results from the atomic scale to specifically developed models at the mesoscopic scale performed during the F-BRIDGE European project between 2008 and 2012. The atomic transport properties of defects and fission gases in uranium dioxide have been extensively studied at the atomic scale, using electronic structure calculations (see reviews by Liu et al. [9] and Dorado et al. [10]) and empirical potential methods (see e.g. Govers et al. [11,12] and Devanathan et al. [13]). This is the first time, however, that such a systematic exercise including the calculation of a wide range of parameters of mesoscale models and the development of such models using the data calculated at the atomic scale was done for a fuel material, even if one only one aspect of in-pile irradiation was considered. In particular, the thermodynamical properties of fuel, the chemical evolution happening during burn-up, or the modification of the mechanical properties were not considered in the investigations presented here.

The outline of the article is as follows. We first present the mesoscale models developed to investigate defects and rare gas behaviour in uranium oxide fuel, and in particular the cluster dynamics and kinetic Monte Carlo methods employed to model the behaviour of defects and fission gases in $\mathrm{UO}_{2}$, as well as the parameters of these models. Second, we describe briefly the atomic scale methods employed, i.e. electronic structure calculations and empirical potential methods. Then, we show the results of the calculation at the atomic scale of the data necessary for the mesoscale models. Finally, we summarise the links built between the atomic and mesoscopic scales by listing the data calculated at the atomic scale which is to be used as input in mesoscale modelling and identifying the gaps.

Many of the calculation results presented in this synthesis have been published individually. When this is the case, the reference is given at the beginning of the section presenting them.

\section{Mesoscale investigation of $\mathrm{UO}_{2}$ under irradiation}

\subsection{Mesoscale modelling}

The modelling of fuel under irradiation at the mesoscopic scale aims at describing the evolution of the fuel microstructure either in-pile or under ion irradiation. In particular, the evolution of the populations of point defects, defect clusters and fission gas atoms and bubbles in one or several grains over macroscopic times are investigated. The knowledge of these quantities is essential since these populations control a large number of material properties and their macroscopic evolution, e.g. nucleation and growth of bubbles and dislocation loops, porosity, re-densification and radiation enhanced diffusion. The mesoscale investigations thus bring further insight into the physico-chemical and mechanical behaviours of fuels, which condition the design and safety of nuclear reactors.

Various types of methods exist to model materials at the mesoscopic scale. Several have been applied to materials, and in particular nuclear fuel under irradiation, such as rate theory methods, kinetic Monte Carlo or phase field methods. The first two types of methods were selected for the multiscale approach developed. These methods and their application to the investigation of the behaviour of defects and gases in $\mathrm{UO}_{2}$ are described in detail in Refs. [14] and [15]. Their principles, the results that can be obtained and the input parameters needed are recalled below.

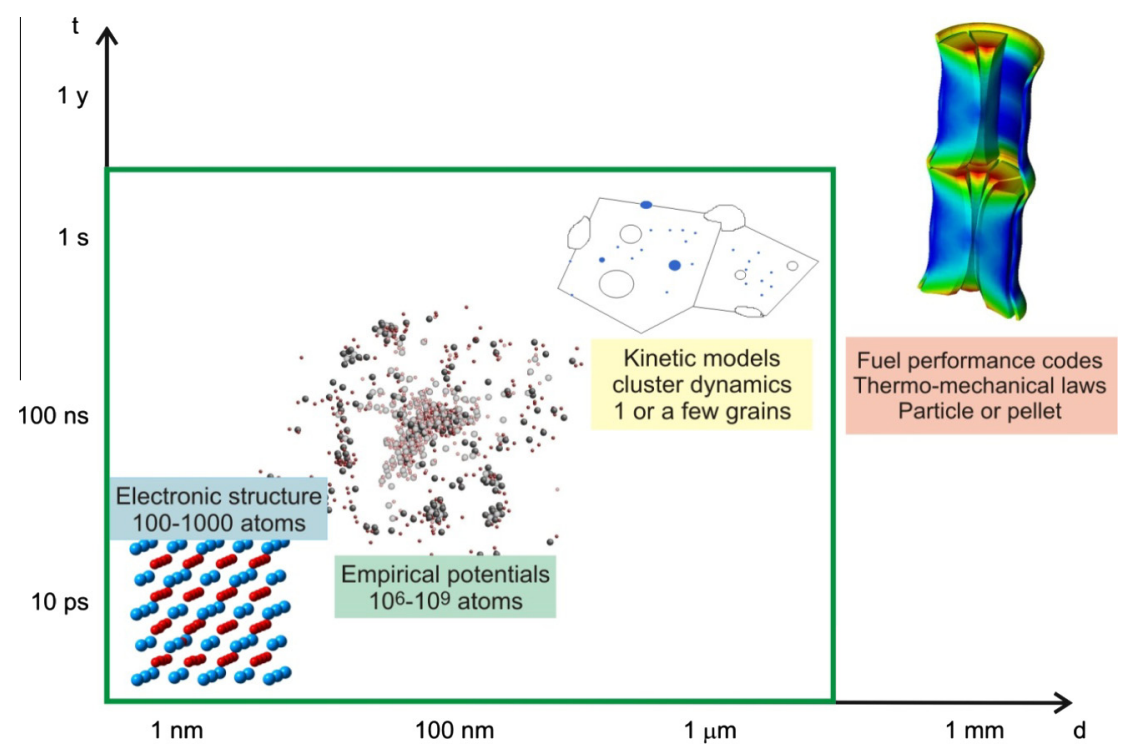

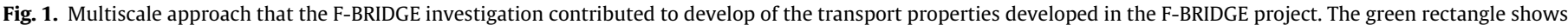

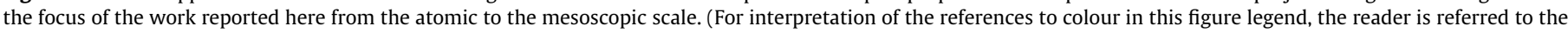
web version of this article.) 


\subsection{Cluster dynamics}

\subsubsection{Principle}

Cluster dynamics is a rate theory method adapted to the simulation of the evolution of point defects and defect clusters in materials [16]. In this method the system investigated is seen as a gas of clusters of various sizes (including monomers) of vacancies, interstitials and solute atoms. The atomic positions are not considered and the properties of the system are often spatially averaged. The evolution of the system is then described by a set of differential equations on species concentrations. In the case of non-conservative clusters consisting of defects (vacancies and self-interstitials) and solute atoms, with a few mobile clusters i.e. that react with or can be emitted by other clusters, the equations can be written as follows:

$$
\begin{aligned}
\frac{d C(n, p)}{d t}= & \sum_{(m, q) \text { mobile }} J_{(n-m, p-q) \leftrightarrow(n, p)}-\sum_{(m, q) \text { mobile }} J_{(n, p) \leftrightarrow(n+m, p+q)} \\
& +G(n, p)-K(n, p)
\end{aligned}
$$

where $C(n, p)$ is the concentration of clusters containing $n$ defects and $p$ solute atoms; $J_{(n, p) \leftrightarrow(m, q)}$ terms are the flux from one cluster size to another; $G(n, p)$ is the source term which describes the production of defects through irradiation; $K(n, p)$ is the sink term which accounts for the annihilation of mobile defects on sinks.

The resolution of these equations yields the concentrations of the various defects and defect clusters over time. In practice, a detailed description is only important for the monomers and small clusters. Approximations are made for large cluster sizes.

\subsubsection{Advantages and limitations}

Due to its low computer cost, cluster dynamics can handle long term evolution that cannot be investigated through atomistic methods. Cluster dynamics enables the development of more general and precise models than standard operational rate theory models previously applied to nuclear fuels [17], which rely on the same physics, but make significant and poorly justified approximations on the cluster size distribution. In comparison, the complete and unbiased description of the cluster size distribution in cluster dynamics is a natural receptacle for the basic parameters yielded by atomic scale studies.

A significant drawback of the method is the loss of space correlations of the elements of the material microstructure which is the price paid for the strong decrease of the computer cost compared to atomistic methods. The improved description compared to earlier rate theory models comes with an increased complexity of numerical resolution. Finally, if cluster dynamics has been extensively applied to metallic systems, developments are still needed for the application to fuels.

\subsubsection{Model developed}

The cluster dynamics method was applied to the study of the behaviour of defects and krypton in irradiated $\mathrm{UO}_{2}$ [15]. The model used for the investigation of fission gas release in $\mathrm{UO}_{2}$ is described in detail in Refs. [14] and [15]. We recall here only the main parameters and assumptions of the model.

Only one lattice composed of neutral $\left(\mathrm{UO}_{2}\right)$ sites is considered in this study. The elementary vacancy (hereafter called V) and interstitial (I) are therefore assumed to be the Schottky defect ( $\mathrm{UO}_{2}$ trivacancy) and the anti-Schottky defect $\left(\mathrm{UO}_{2}\right.$ tri-interstitial), respectively. Both types of defects are assumed to be mobile and can therefore react with other defects and be absorbed or emitted by clusters. The gas atoms are supposed to be mobile in two configurations, as interstitial (noted $\mathrm{Kr}$ ) or combined with a di-Schotthy vacancy $\left(\mathrm{Kr}-\mathrm{U}_{2} \mathrm{O}_{4}\right.$ complex noted $\mathrm{V}_{2} \mathrm{Kr}$ ), as may occur in metals.
The energetic and kinetic parameters of the model were taken from atomic scale calculations (see Sections 2.4 and 6). The source term is evaluated on the basis of classical molecular dynamics simulations [18]. The cascade resulting from the implantation of a single ion ( $250 \mathrm{keV}$ krypton ion or fission product) is assumed to break down into lower energy independent subcascades of $\sim 20 \mathrm{keV}$, each producing on average one cavity of 40 vacancies, $\sim 2.7$ loops of 15 interstitials and 40 additional isolated interstitials and vacancies. Krypton is assumed to be inserted in an interstitial position during implantation.

\subsubsection{Results obtained}

The fraction of $\mathrm{Kr}$ reaching the surface and released at $1250{ }^{\circ} \mathrm{C}$ was calculated and compared to the experimental data, as shown on Fig. 2.

The results of the cluster dynamics simulations enabled us to propose two mechanisms for krypton release: $8 \%$ of the initially interstitial krypton immediately reaches the surface (initial burst) while the remaining fraction is trapped by vacancies to form successively $\mathrm{VKr}$ ( $\mathrm{Kr}$ in a Schottky defect) and $\mathrm{V}_{2} \mathrm{Kr}$ ( $\mathrm{Kr}$ in a di-Schottky defect) and is released during a second stage following a quasi Fickian behaviour. Eventually, $11 \%$ of the krypton is released. A Fickian analysis of the release curve of this second stage yields a diffusion coefficient of $\mathrm{V}_{2} \mathrm{Kr}$ of $4 \times 10^{-21} \mathrm{~m}^{2} / \mathrm{s}$. A preliminary sensitivity study of the release as a function of the vacancy migration energy showed that more mobile vacancies induce a more effective $\mathrm{Kr}$ bulk trapping, which leads to a lower initial release. Additional investigations, however, are necessary to confirm these results and determine the precise mechanisms at work.

Although the initial fission gas release cannot be accounted for by a Fickian behaviour, the second stage can be fairly well described by a purely diffusive process, which means this kind of experiment is a relatively simple but reliable tool to evaluate diffusion coefficients. Cluster dynamics enabled a comprehensive interpretation of both stages.

Models taking into account grain boundaries to describe polycrystalline $\mathrm{UO}_{2}$ must still be developed to improve the description of the fission gas behaviour.

\subsection{Kinetic Monte Carlo}

\subsubsection{Principle}

Kinetic Monte Carlo (KMC) methods [19-24] are stochastic methods enabling the simulation of the time evolution of a system when the processes governing the evolution are known. The scope

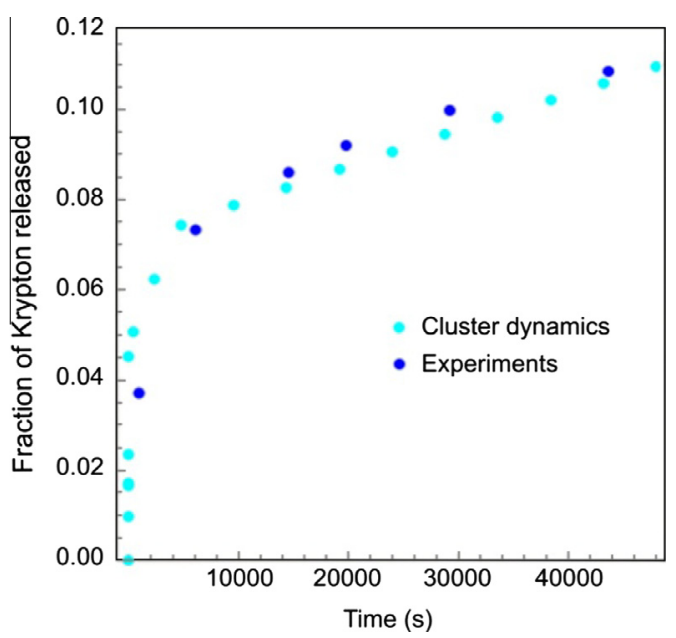

Fig. 2. Simulated and experimental fraction of $\mathrm{Kr}$ released for the best parameter set [15]. 
of applications for KMC is extraordinarily wide, ranging from epidemiology and population kinetics to surface growth or radiation damage. Three commonly used kinetic Monte Carlo models are:

- Lattice or atomistic KMC $[25,26]$ : In this variant the system is the atomic lattice, the processes of evolution are jumps of the atoms composing the system from a position to another and the jump rate is due to local environment.

- Object KMC [27-29]: Only the positions of the "objects" e.g. defects or impurities are considered, and the positions of atoms on the background lattice are not included in the simulation. This is the kind of model applied to $\mathrm{UO}_{2}$ in F-BRIDGE.

- Event KMC [30] is a variant of object KMC where events are related to interactions between objects and not to atomic jumps on the lattice.

A kinetic Monte Carlo simulation consists of determining at every time step a plausible subsequent state of the system through the selection of a transition among all the possible transitions according to their occurrence probability law. A large number of steps are simulated so that the average result can be evaluated. In lattice KMC, one must choose at every time step an event among all the possible jumps and keep track of the successive positions of the mobile atoms of the system. From a technical point of view there are two problems to solve at each step: selecting an appropriate event and determining the time at which it occurs. The transition events are chosen randomly proportionally to their transition rates. Once a transition is chosen, its time of occurrence is determined by generating a random number and comparing it with the probability that the system undergoes its current transition at a given time. At the end of the simulation, various interesting parameters can be extracted, such as diffusion coefficients or thermodynamic constants.

\subsubsection{Advantages and limitations}

Kinetic Monte Carlo provides a methodology to overcome the molecular dynamics time limitation. The main advantage of KMC methods is that systems out of thermodynamic equilibrium can be simulated for microscopic time scales while keeping spatial correlations. In addition, transition events can be turned off or on to separate the effects.

All possible events and their rates, however, must be known in advance, the method in itself is not predictive. KMC are also computationally expensive, in particular compared to rate theory methods. In the case of plain lattice KMC, calculation time scales linearly with the number of atoms.

\subsubsection{Results obtained}

A simple kinetic Monte Carlo model was applied to the investigation of the migration of oxygen interstitials in $\mathrm{UO}_{2}$. Two cases were considered:

- In the first case, only mono-interstitials were able to move and they could only move into an interstitial site if that site was empty. Mono-interstitials were also prevented from occupying nearest neighbour sites because of the repulsion observed in electronic structure results.

- In the second case di-interstitials could form with a rate governed by the calculated binding energy and interstitial diffusion barrier and then hop via a di-interstitial mechanism. Clusters containing more than two interstitials were prevented from forming via a blocking scheme equivalent to the one used for mono-interstitials in the first case. The influence of the barrier to the di-interstitial dissociation was investigated by considering two different barriers: one calculated in GGA which is slightly lower than the di-interstitial migration energy [31] and one obtained during the project using GGA $+\mathrm{U}$, which is higher than the di-interstitial migration energy (di-interstitial models 1 and 2, respectively).

The evolution of the oxygen diffusivity as a function of the deviation from stoichiometry $x$ in the hyperstoichiometric regime yielded by the "mono-interstitial" and the two "di-interstitial" models at $1073 \mathrm{~K}$, as well as the experimental diffusion coefficients of tracer ${ }^{18} \mathrm{O}$ obtained by mass spectrometry or nuclear reactions [32,33], are shown in Fig. 3.

It can be seen that in the mono-interstitial model $D$ initially increases with the deviation from stoichiometry until the interstitials begin to interact with each other by blocking available diffusion paths, which results in decreasing $D$ for high $x$. It is seen that the oxygen diffusivity calculated by this simple blocking model is in good agreement with experimental observations at low $x$ but is much lower than the maximum $D$ observed experimentally for $x=0.12$.

The inclusion of the di-interstitial mechanism increases the oxygen diffusivity for all deviations from stoichiometry, especially at high $x$ values, which greatly improves the agreement with the experimental data. The oxygen diffusivity is higher in the second di-interstitial model since it encourages the formation of more di-interstitials, which are more mobile than mono-interstitials. This model seems to agree the best with the experimental data. It is important to note, however, that the experimental diffusivities exhibit a very large scatter, and that more precise experimental data would be needed to discriminate between the two di-interstitial models.

\subsection{Input parameters of mesoscale models}

Mesoscale models, in particular cluster dynamics and Kinetic Monte Carlo, employ a certain number of parameters to describe the behaviour of systems under irradiation. One can distinguish the data concerning the thermal evolution of the material, its defects and the fission products and the data on phenomena induced by irradiation. The main parameters of the mesoscale models applied in the F-BRIDGE project to the transport properties of defects and rare gases are as follows.

\subsubsection{Thermal evolution}

- Formation, migration, binding energies of point defects

- Formation, migration, binding energies of defect clusters in bulk as a function of size

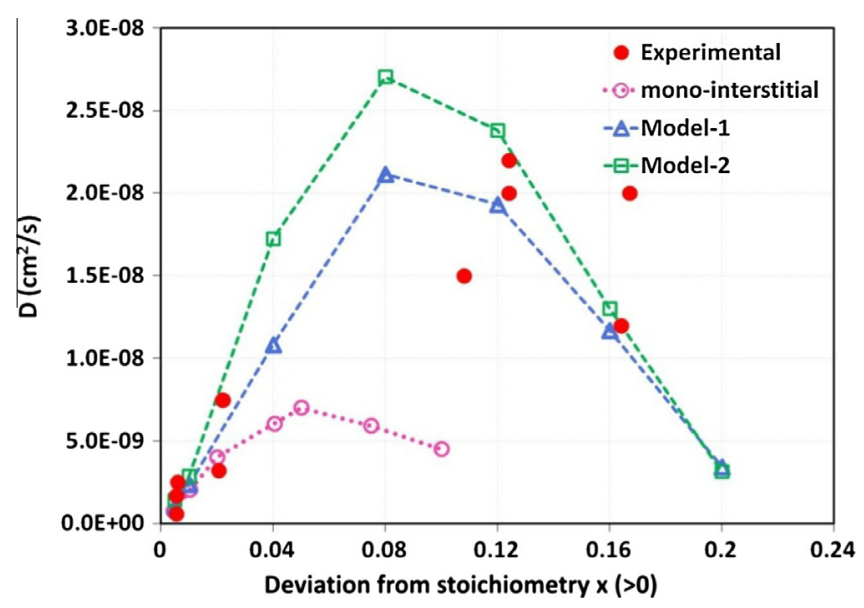

Fig. 3. Oxygen diffusivity $D$ as a function of the deviation from stoichiometry $x$ in the hyperstoichiometric regime at $1073 \mathrm{~K}$ for the various models, as well as the experimental data. 
- Migration energies near extended defects and grain boundaries

- Incorporation and migration energies of solute atoms (isolated or clusters) in bulk and near extended defects

- Equation of state for solute

- Surface tension of solute clusters/bubbles

- Recombination/elimination rates of defects on sinks.

\subsubsection{Irradiation induced phenomena}

- Damage created by irradiation: source term

- Volume evolution of extended defects during irradiation

- Irradiation induced resolution of defects or solute atoms

- Precipitation of solutes

- Radiation induced and enhanced diffusion

As will be shown in Sections 5 and 6 of this article, this data can be determined at the atomic scale.

\section{Atomic scale methods used to calculate the input parameters of mesoscale models}

Two types of methods were used to calculate at the atomic scale the input parameters of mesoscale models: electronic structure methods and empirical potentials. These methods are described extensively in the literature, for instance in Refs. [34,35,36]. We give below a short description of the two types of methods.

\subsection{Electronic structure calculations}

The properties of matter can be deduced from calculations at the atomic scale by solving the Schrödinger equation. This approach, which considers the interactions between the nuclei and the electrons described by a set of coupled differential equations, and necessitates only little prior knowledge of the system studied or parameterization, is called electronic structure or $a b$ initio. Only an approximated solution to the many body problem can be obtained using numerical techniques, for instance the density functional theory (DFT).

In this framework the total energy of a system is calculated as a function of its electronic density. This reduces significantly the number of variables of the problem compared to other quantum chemistry methods, and therefore the computational cost needed to solve approximately the Schrödinger equation. The exact analytical expression for the energy as a function of the electronic density is unknown, the interactions impossible to calculate exactly are gathered in a term called the exchange-correlation term. This separation is justified by the fact that this part of the energy, even if it is important for the chemical bonding, is small compared to the total energy of the system. Various approximations were proposed for the exchange-correlation term, for instance the local density (LDA) or generalised gradient (GGA) approximations, or for strongly correlated systems, such as actinide compounds, the DFT + U method or hybrid functionals.

\subsection{Empirical potentials and classical molecular dynamics}

Empirical potentials constitute the second large family of atomic scale modelling methods. In contrast to quantum mechanical methods, empirical pair potentials parameterise the interaction between atoms in terms of a simple analytical function. Therefore, the complex and computationally demanding description of the electron dynamics is withdrawn in favour of a simpler effective model.

Pair potentials, depending only on the distances between the atoms of the system, and usually containing a short-range repul- sion, a van der Waals attraction, a long-range Coulomb interaction and a covalent bonding term, are very commonly-used potentials, especially for mostly ionic compounds such as $\mathrm{UO}_{2}$. The parameters of these potentials are determined empirically by fitting the free parameters to a set of thermophysical properties obtained experimentally or from electronic structure calculations.

The polarisability of the constitutive ions is a significant contribution to the total energy of the system. The most common treatment to take into account this polarisability, called the rigid ion model, is to combine the effects of polarisability of the ions into the attractive part of the short range interaction potential. A more complex method is the core-shell model, in which each ion is represented as a positively charged, massive core coupled by a harmonic spring to a negatively charged shell, massless or exhibiting a small mass.

Different sets of potential parameters were proposed as suitable for modelling the bulk and defect behaviour of nuclear oxide fuels [36]. These potentials are very often combined with the simulation of molecular dynamics, which enables the simulation of the evolution in time of a system and to explore its configuration space. This combination is referred to as classical molecular dynamics (CMD).

\section{Assessment of atomic scale methods for the description of bulk and defective $\mathrm{UO}_{2}$, as well as rare gas behaviour}

Simulation of the irradiation behaviour of $\mathrm{UO}_{2}$ is one of the most challenging problems for atomic scale modelling because of the particular properties of actinide compounds and of the complexity of the processes induced by irradiation. During the FBRIDGE project comprehensive work was done to review and improve the state of the art of the atomic scale description of $\mathrm{UO}_{2}$ under irradiation, in particular the description of perfect and defective $\mathrm{UO}_{2}$, as well as of the behaviour of rare gases, using electronic structure and empirical potential methods [36]. The results of the assessment done are summarised here.

The evaluation of the density functional theory performed $[36,10]$ shows first that the GGA approximation yields reasonable properties of bulk $\mathrm{UO}_{2}$ (except for the electronic structure), but that the description of the defect behaviour is only qualitative. The GGA + U formalism combined to a careful monitoring of the orbital occupancies (see Section 5.1.1) improves significantly the description of bulk $\mathrm{UO}_{2}$ and of oxygen transport. This is in agreement with the conclusions of Liu et al. [9]. Precise experiments on uranium diffusion similar to the recent ones performed on oxygen are needed to complete the assessment on uranium defects. Other promising methods like hybrid functionals should also be assessed.

The validity of several types of functionals for the description of bonds formed by rare gases has also been assessed on model molecular compounds [37]. The results show that when the rare gas atom shares density with the neighbouring atoms, the GGA functionals yield good geometries and qualitatively correct binding energies, even if these are quite significantly overestimated. The use of hybrid functionals enables us to obtain good geometries and satisfactory binding energies. For compounds in which the rare gas atom forms weak dispersive-like bonding, the accuracy yielded by the various functionals is not as good. No functional gives satisfactory binding energies of all the association compounds investigated. Several GGA and hybrid functionals yield correct geometries, even if some isomers are not obtained. One GGA functional (PBE) yields qualitatively correct results for the compounds of the three rare gases and several hybrid functionals give satisfactory energies for He compounds. The results of this study must be confirmed on more solid systems, for instance small clusters simulating the first coordination sphere of the rare gas incorporated in a material. 
The assessment of the empirical potentials available for $\mathrm{UO}_{2}$ shows that despite the lack of a mechanism for charge transfer the general ordering of defect energies is well respected. The comparison of the migration energies yielded by empirical potentials with experimental and DFT $+U$ results is surprisingly consistent, even if significant scatter is observed in the results between different pair potentials. Moreover, these empirical potentials enable a correct description of many of the important aspects of radiation damage. For example, simulations using these potentials reproduce the initial displacement cascade, the molten core region at the centre of the cascade and the final recombination phase of the irradiation period. The use of core-shell potentials is desirable as they offer an accurate and simple method of describing a material dielectric relaxation that may be important for correctly obtaining point defect interaction and formation energies. But there are technical and conceptual difficulties in their use for the simulation of displacement cascades [38]. It remains to be seen whether these difficulties can be overcome, or whether the coreshell models will not be suitable for the specific case of radiation damage simulations. Potentials allowing one to take into account charge transfer would also be beneficial for the study of $\mathrm{UO}_{2}$ under irradiation.

In addition, various static methods of exploration of potential energy surfaces have been checked against molecular dynamics and experimental results. The results show that despite some slight discrepancies, the agreement between static methods and molecular dynamics values is generally satisfactory.

Finally, the question of the validity of the simulation of radiation damage using displacement cascades has been investigated. This question remains essentially open given the difficulty in simulating radiation damage, especially in insulators. Further developments and electronic structure calculations would be desirable for validation of the far-from-equilibrium configurations formed in these simulations, in particular for short ion-ion distances. Moreover, the electronic part of irradiation damage in ionic fuel materials is currently not included in these simulations, but is an important part both of the overall stopping process and of the transfer of energy to the matrix. New developments are currently in progress to describe the electronic effects in metals and insulators.

\section{Investigation of the transport properties in $\mathrm{UO}_{2}$ at the atomic scale}

We present here the results of the atomic scale calculations of the data necessary for the mesoscale models (see Section 2.4). First, the results of the investigations of the behaviour of defects are shown. This includes the calculation of the formation, migration, binding and recombination energies, as well as the investigation using the simulation of displacement cascades and thermal spikes of the damage created by irradiation to determine the source term of the mesoscale models. The radiation induced diffusion has also been considered. Second, the studies of the behaviour fission gases and helium in $\mathrm{UO}_{2}$ are presented. In particular, the results on the incorporation and migration of gases in $\mathrm{UO}_{2}$, as well as on the formation of bubbles and the resolution under irradiation are shown.

\subsection{Transport properties of defects in $\mathrm{UO}_{2}$}

\subsubsection{Electronic structure study of $O$ and $U$ defect behaviour}

The formation energies of various point defects in $\mathrm{UO}_{2}$ were calculated in the framework of the density functional theory in the DFT + U formalism with the orbital control scheme developed during the F-BRIDGE project to ensure the convergence to the electronic ground state of the system [39-41]. Neutral supercells were considered. The $U$ and $J$ parameters of the DFT $+U$ approximation were not fitted, but the $4.50 \mathrm{eV}$ and $0.51 \mathrm{eV}$ values determined experimentally by Kotani and Yamazaki [42] using analysis of X-ray photoemission spectra were used. Because of the prohibitive cost associated to the modelling of paramagnetic systems in DFT $+\mathrm{U}$, this magnetic order is approximated by a collinear anti-ferromagnetic order.

The point defects as follows were considered: $\mathrm{O}$ and $\mathrm{U}$ mono-interstitials, $\mathrm{O}$ and $\mathrm{U}$ mono-vacancies, $\mathrm{O}_{2}$ di-interstitials, Schottky defects $\left(\mathrm{UO}_{2}\right.$ trivacancies) and Frenkel pairs. For the defects involving more than one atom, infinitely separated and bound configurations were investigated. Two $\mathrm{O}$ di-interstitial bound configurations and the three non-equivalents configurations of the Schottky defects were considered. The formation energies obtained relative to metallic uranium and gaseous dioxygen are listed in Table 1 [43].

The analysis of the defect formation energies presented in Table 1 shows that the formation of the oxygen interstitial, the bound Schottky defects and di-interstitials (especially in the configuration where 3 oxygen atoms are split over one site) is very favourable. The binding energy of Schottky defects amounts to several $\mathrm{eV}$, as can be seen by comparison with the formation energy of isolated Schottky defects.

The analysis of the charge redistribution induced in the lattice by the point defects shows the creation of $\mathrm{U}^{5+}$ or $\mathrm{U}^{3+}$ cations for charge compensation. The oxygen interstitial induces the formation of two $\mathrm{U}^{5+}$ cations, the oxygen vacancy the formation of two $\mathrm{U}^{3+}$ cations and the uranium vacancy the formation of four $\mathrm{U}^{5+}$ cations. The position of the $\mathrm{U}^{5+}$ and $\mathrm{U}^{3+}$ cations relative to the point defect has an influence on the formation energy of the defect and has to be carefully monitored.

Since the end of the F-BRIDGE project several studies [44-46] focused on the various possible charged states of the point defects in uranium dioxide and showed that $\mathrm{UO}_{2}$ is strongly ionic and that the large majority of defects formed in this materials are charged.

The migration energies of several elementary migration mechanisms for oxygen and uranium defects were also investigated in the DFT [47], and DFT + U framework [48,49] using the nudged elastic band method (NEB) [50]. The migration mechanisms considered are as follows:

- Vacancy mechanisms, in which an atom moves to a nearest neighbour vacancy of the same chemical specie in the $\langle 100\rangle$ or the $\langle 110\rangle$ directions. In the case of uranium simple mechanisms, as well as a combined mechanism involving movement of the $U$ vacancy and of the neighbour oxygen atoms were considered.

Table 1

Formation energies of point defects in $\mathrm{UO}_{2}$ yielded by DFT $+\mathrm{U}$.

\begin{tabular}{lc}
\hline Point defect & Formation energy $(\mathrm{eV})$ \\
\hline Oxygen interstitial $I_{O}$ & -0.05 \\
Uranium interstitial $I_{U}$ & 5.36 \\
Oxygen vacancy $V_{O}$ & 5.38 \\
Uranium vacancy $V_{U}$ & 10.43 \\
Bound di-interstitial $\left(I_{O O}^{O}\right)$ & 0.43 \\
Bound di-interstitial $\left(I_{O O}^{X}\right)$ & -0.26 \\
Bound Schottky defect $S_{1}$ & 3.32 \\
Bound Schottky defect $S_{2}$ & 2.54 \\
Bound Schottky defect $S_{3}$ & 2.82 \\
Infinitely separated Schottky defect $S^{*}$ & 10.66 \\
Infinitely separated Oxygen Frenkel pair $\mathrm{FP}_{O}^{*}$ & 5.33 \\
Bound uranium Frenkel pair FP & 11.18 \\
Infinitely separated uranium Frenkel pair $\mathrm{FP}_{U}^{*}$ & 15.79 \\
\hline
\end{tabular}


- A direct interstitial mechanism, in which an interstitial atom in an octahedral site moves to the next nearest octahedral interstitial site.

- An indirect interstitial mechanism (also called interstitialcy), in which an atom in an octahedral site replaces an atom on a neighbouring lattice site, which in turn moves to an octahedral interstitial site.

The migration energies obtained for the various mechanisms investigated are shown in Table 2 . The results of these calculations enable us to determine the precise mechanisms for the $\mathrm{O}$ and $\mathrm{U}$ migration. In the presence of interstitials, $\mathrm{O}$ and $\mathrm{U}$ migrate through an indirect mechanism, also called interstitialcy mechanism. In presence of vacancies, the migration occurs through jumps in the 100 direction, but for uranium, this migration is combined with significant movement of the nearest neighbour oxygen atoms.

\subsubsection{Metadynamics investigation of 0 self-diffusion}

To study longer-term evolution, the self-diffusion of oxygen and uranium atoms in pristine $\mathrm{UO}_{2}$ was also simulated using the Lagrangian metadynamics method [51,52] implemented in the CP2K program package [53] combined to empirical potentials.

The implementation of rigid-ion and core-shell models into CP2K performed in the framework of the F-BRIDGE project allowed the combination and extension of these new functionalities with features already implemented in $\mathrm{CP} 2 \mathrm{~K}$. In this way, it was straightforward to build a link to the metadynamics module of $C P 2 \mathrm{~K}$. Metadynamics is an accelerated sampling method allowing for the fast exploration of a system's free energy landscape, even if energy barriers are present which are large compared to the typical thermal fluctuations. In this way, rare events can be observed within the time periods accessible by standard molecular dynamics simulation runs. The free energy landscape of a reaction path is reconstructed from the normal evolution of a system biased by a history-dependent potential which is obtained from a sum of Gaussians functions centred along the trajectory based on a suitably chosen set of collective variables. Metadynamics simulations can also be employed for a brute-force exploration of the free energy surface to spot all nearby minima. Thereafter, the NEB method or any similar technique is applied for the refinement of the reaction path.

In the case of the self-diffusion of oxygen and uranium in $\mathrm{UO}_{2}$ a suitable and obvious collective variable to drive the system in phase space is the displacement of a target atom from its original position in the lattice. The history-dependent bias potential discourages the target atom to visit the same spot in the lattice repeatedly. Such a collective variable was implemented into the metadynamics module of $\mathrm{CP} 2 \mathrm{~K}$.

Two types of empirical potentials, a rigid-ion [54] and a coreshell [55] model, were employed for the simulations to study the

Table 2

Migration energies of point defects in $\mathrm{UO}_{2}$ yielded by DFT and DFT $+\mathrm{U}$.

\begin{tabular}{|c|c|c|c|}
\hline & \multirow[t]{2}{*}{ Mechanism } & \multicolumn{2}{|c|}{$\begin{array}{l}\text { Migration } \\
\text { energies (eV) }\end{array}$} \\
\hline & & DFT & $\mathrm{DFT}+\mathrm{U}$ \\
\hline \multirow[t]{4}{*}{ Oxygen } & Direct interstitial & 3.6 & 3.2 \\
\hline & Indirect interstitial & 1.1 & 0.9 \\
\hline & Vacancy in $\langle 100\rangle$ direction & 1.2 & 0.7 \\
\hline & Vacancy in $\langle 110\rangle$ direction & 2.7 & 2.5 \\
\hline \multirow[t]{5}{*}{ Uranium } & Direct interstitial & 5.8 & 7.9 \\
\hline & Indirect interstitial & 1 & 4.1 \\
\hline & Vacancy in $\langle 100\rangle$ direction & 1 & 7.2 \\
\hline & Vacancy in $\langle 110\rangle$ direction & 4.4 & 5.5 \\
\hline & $\begin{array}{l}\text { Vacancy in }\langle 100\rangle \text { direction combined with } 0 \\
\text { displacement }\end{array}$ & & 3.6 \\
\hline
\end{tabular}

impact of the potential type on the diffusion behaviour. Metadynamics was used for the exploration of the free energy surface starting from an equilibrated sample of $5 \times 5 \times 5$ unit cells of $\mathrm{UO}_{2}$. The displacement of the target oxygen or uranium atom with respect to its original lattice position was used as the only collective variable. Already after less than 100 picoseconds of simulation time various migration events involving activation energies of several eV were observed. Fig. 4 shows the free energy profile for the self-diffusion of an oxygen atom at $1500 \mathrm{~K}$ and 1 bar in pristine $\mathrm{UO}_{2}$ after about $100 \mathrm{ps}$ simulation time.

A strong dependence of the free energy profile on the simulation temperature and the applied potential is observed. The core-shell potential AMD simulations show already for lower temperatures $(500-1000 \mathrm{~K})$ a relatively flat free energy profile indicating an enhanced diffusivity of oxygen. The oxygen atom quickly leaves its original lattice position to explore the nearby interstitial octahedral holes at a distance of about $4 \AA$ A. Energy barriers of up to $2 \mathrm{eV}$ are surpassed during the migration processes which gives access to simulation time scales much beyond any standard molecular dynamics simulation. These preliminary results are encouraging and electronic structure based metadynamics simulations of transport phenomena in $\mathrm{UO}_{2}$ seem to be in reach.

\subsubsection{Classical molecular dynamics investigation of $O$ and $U$ self- diffusion near grain boundaries}

To take into account the polycrystalline nature of materials, the influence of grain boundaries on self-diffusion was also studied. The uranium and oxygen self-diffusion near two symmetrical grain boundaries with different misorientation angles between grains the tilt $\Sigma 5$ and $\Sigma 41$ - were investigated [56,57] over temperatures varying from $300 \mathrm{~K}$ to $2100 \mathrm{~K}$ using molecular dynamics simulations and the Morelon empirical potential [54]. The $\Sigma 41$ grain boundary exhibits a small misorientation angle and the interface defects correspond to edge dislocations without any significant space between both mono-crystals. The $\Sigma 5$ grain boundary exhibits a larger misorientation $\left(36.9^{\circ}\right)$. This induces a gap of about $1 \AA$ A between the two mono-crystals which can be seen as a line of Schottky defects at the interface [58].

Since the atomic structure near the grain boundaries changes continuously as a function of the distance from the interface [33], the diffusion properties are likely to change too. In order to study the influence of grain boundary structure on self-diffusion coefficients the simulation box was divided in $3 \AA$ Aide slabs along

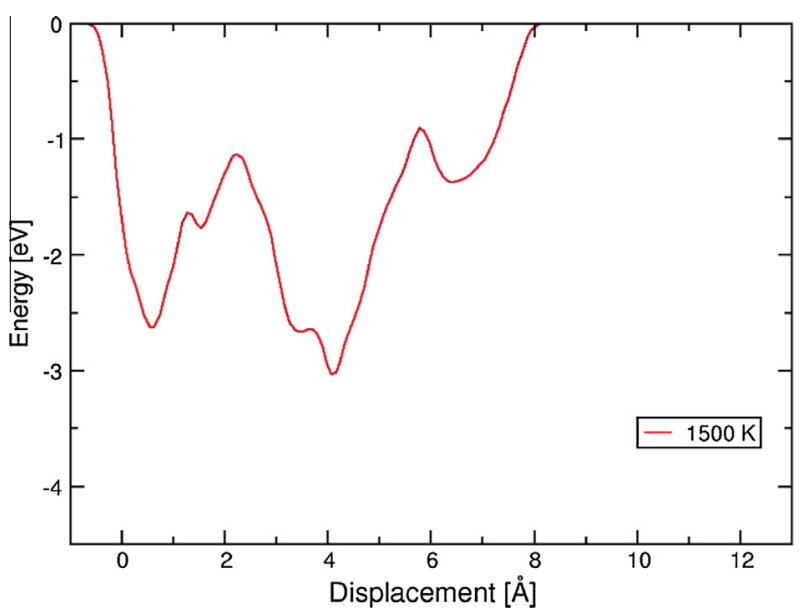

Fig. 4. Free energy profile after 100 ps of metadynamics simulation using a rigidion potential for the migration of an oxygen atom at $1500 \mathrm{~K}$ and $1 \mathrm{bar}$ in pristine $\mathrm{UO}_{2}$. Zero refers to the original interstitial position of the $\mathrm{O}$ atom. The minima correspond to the next octahedral hole positions in the $\mathrm{UO}_{2}$ lattice. 
the axis parallel to the grain boundary plane. For each slab a selfdiffusion coefficient was calculated by averaging the mean square displacement of all the atoms present in the slab. Fig. 5 shows the evolution of the oxygen self-diffusion coefficients at $1500 \mathrm{~K}$ with the distance to the interface for the $\Sigma 41$ and $\Sigma 5$ grain boundaries. For comparison the bulk self-diffusion coefficient obtained in single-crystal is displayed in the same graph (blue horizontal line).

Both grain boundaries exhibit similar behaviours and three zones can be distinguished according to the distance from the interface. In the zone closest to the interface, called grain boundary core, the diffusion coefficients are highest and relatively constant. This zone is narrow: approximately $12 \AA$ in width (twice $6 \AA$ ). For distances from the interface ranging from 6 to $20 \AA$, i.e. in the intermediate zone, the diffusion coefficients decrease strongly. Then, for distances greater than $20 \AA$, the diffusion coefficients decrease slightly. At $50 \AA$ from the interface the diffusion coefficients are 40 times and 60 times lower than the values at the interface for $\Sigma 41$ and $\Sigma 5$, respectively. This is a significant drop, but all coefficients are still two orders of magnitude higher than those calculated in the bulk. This tends to prove that grain boundaries do not act as defect sinks but enhance diffusion.

The evolution of self-diffusion coefficients with temperature in the vicinity of $\Sigma 41$ and $\Sigma 5$ grain boundaries was studied for temperatures ranging from $300 \mathrm{~K}$ to $2100 \mathrm{~K}$ to determine the activation energy to the self-diffusion, and subsequently the migration energies of the $\mathrm{O}$ and $\mathrm{U}$ defects. Since uranium and oxygen self-diffusion coefficients are constant in the grain boundary core, calculations were performed inside this zone.

The migration energies resulting from the Arrhenius diagrams of the diffusion coefficients in the grain boundaries are presented in Table 3 and compared with those of the bulk.

It can be seen from Table 3 that the uranium migration energies obtained near the grain boundaries, approximately $0.8 \mathrm{eV}$, are much lower than the point defect migration energies calculated in near-stoichiometric bulk $\mathrm{UO}_{2}$ using electronic structure calculations $(4.1 \mathrm{eV}$ for the interstitial and 3.6 for the vacancy, see Section 5.1.1) or measured experimentally in polycrystalline $\mathrm{UO}_{2}$ matrices (3.03-4.68 eV [33]). This result confirms that grain boundaries considerably enhance the migration of uranium atoms. This is in agreement with the experiments carried out by Sabioni et al. [59] who measured an increase of five orders of magnitude for the uranium self-diffusion coefficient near a grain boundary compared with the one in a single-crystal.

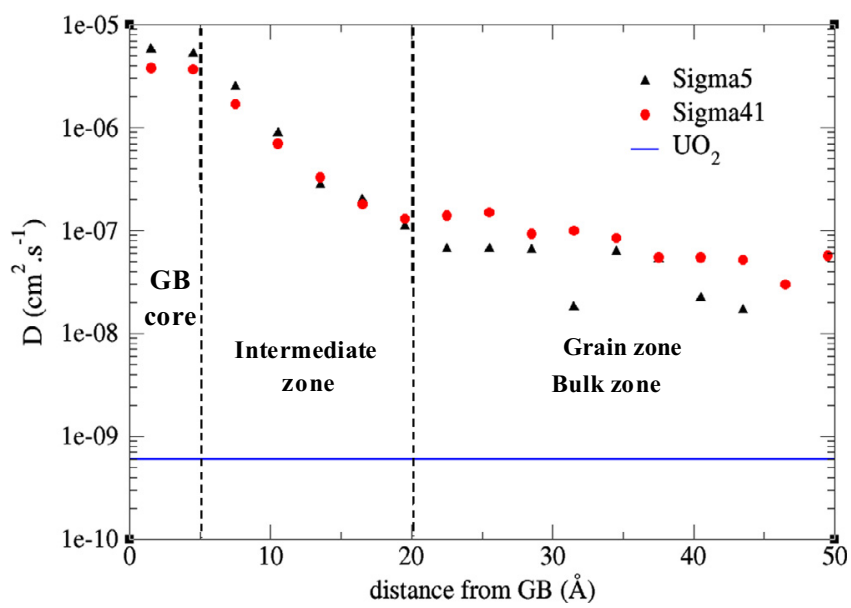

Fig. 5. Oxygen self-diffusion coefficients as a function of the distance from the grain boundary. The horizontal blue line corresponds to the diffusion coefficient in bulk $\mathrm{UO}_{2}$ [56]. (For interpretation of the references to colour in this figure legend, the reader is referred to the web version of this article.)
Table 3

Migration energies (in $\mathrm{eV}$ ) resulting from the fit of the Arrhenius diagram of the diffusion coefficients in the grain boundaries and comparison with the bulk values. The two values for the bulk correspond to the various mechanisms (vacancy/ interstitial migration).

\begin{tabular}{lllll}
\hline & $\Sigma 5$ & $\Sigma 41$ & Bulk Morelon & Bulk DFT + U (Section 5.1.1) \\
\hline $\mathrm{O}$ & 0.6 & 0.8 & $0.3 / 0.7$ & $0.7 / 0.9$ \\
$\mathrm{U}$ & 0.8 & 0.8 & $3.9 / 4.2$ & $3.6 / 4.1$ \\
\hline
\end{tabular}

Even if there is also an apparent enhancement of the $\mathrm{O}$ diffusion near the GB, the oxygen migration energies in the two types of grain boundaries are close to the ones obtained in the bulk (near stoichiometric or hyperstoichiometric) using the Morelon potential or in DFT + U (see Table 3). This agrees well with the fact that no difference is seen in the oxygen self-diffusion between single crystalline and polycrystalline $\mathrm{UO}_{2}[60,48]$. The small size of the systems and the relatively short times simulated could be responsible for the difference in the experimental and simulated diffusion coefficients.

In both types of grain boundaries, diffusion has been found to be anisotropic. The diffusion is significantly faster along the grain boundary interfaces than perpendicular to the grain boundary plane. Two different mechanisms for the oxygen atoms were found for the two GBs. For $\Sigma 41$ oxygen diffusion occurs through hopping mechanisms in the three directions, whereas in $\Sigma 5$, we observe a hopping mechanism along the grain boundary interface and a continuous (Brownian) diffusion in the direction perpendicular to the interface. The precise migrating species remain, however, to be determined.

\subsubsection{Empirical potential study of oxygen and uranium Frenkel pair recombination}

An important phenomenon occurring under irradiation and determining the final quantity of defects created is the recombination of defects. The oxygen and uranium Frenkel pair (FP) recombination mechanisms were studied in $\mathrm{UO}_{2}$ [61] using a dynamical core-shell empirical interatomic potential, which enables one to take into account the polarisability of the ions. The results were compared to a more conventional rigid-ion model. The analysis of the simulation performed indicates that the FP recombination is a complex process involving several phenomena. The FP recombination can happen instantaneously when the distance between the interstitial and the vacancy is small (1st and 2nd neighbours for oxygen, 1 st to 3rd neighbours for $U$ ) and is thermally activated at larger separation distances. Other criteria, however, can prevail over the interstitial-vacancy distance. The surrounding environment of the FP defect, the mechanical stiffness of the matrix and the orientation of the migration path are shown to be major factors acting on the FP lifetime.

Fig. 6 shows a schematic sketch of the FP recombination paths investigated for the oxygen. Several simulations starting from different initial configurations were run at various temperatures.

The results of these simulations are collected in Fig. 7 as Arrhenius plots from which the average lifetimes of a FP can be derived. The straight lines in this figure are the best fits using an Arrhenius function.

$\tau=\tau_{0} \exp \left(E_{a} / k_{B} T\right)$

where $E_{a}$ is the recombination activation energy or energy barrier that an interstitial must overcome to recombine with the vacancy, and $\tau_{0}$ is a pre-exponential factor.

The simulation results show that core-shell and rigid-ion models provide a similar qualitative description of the FP recombination mechanisms. However, the FP stabilities determined by both models significantly differ in the lower temperature range consid- 
(a) $1^{\text {st }}$

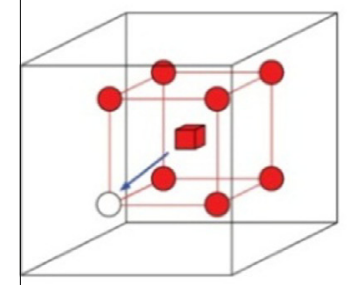

(b) $2^{\text {nd }}$

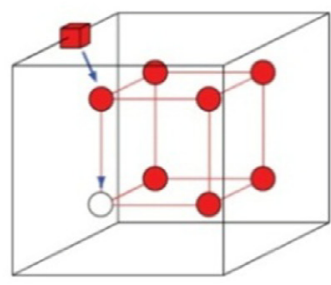

(d) $4^{\text {th }} \quad$ type II

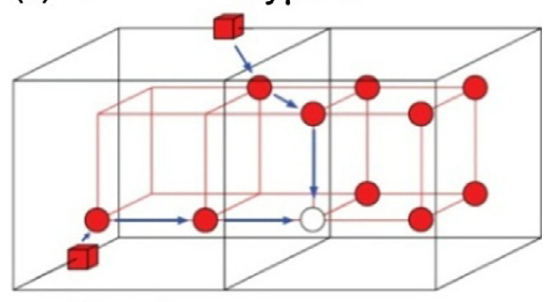

type I (c) $3^{\text {rd }}$

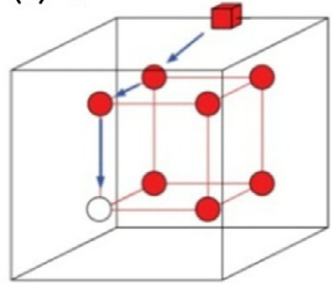

(e) $5^{\text {th }}$

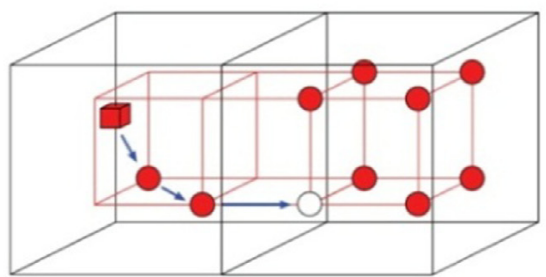

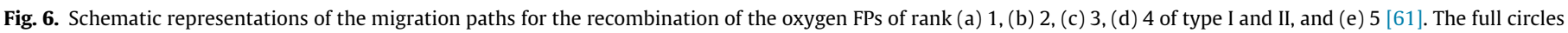
represent the oxygen atoms on a regular lattice site, the empty circles the vacancies and the full squares the interstitials.

ered. The recombination time of the oxygen FPs can be up to an order of magnitude lower in the core-shell model at $T=600 \mathrm{~K}$. These differences highlight the importance of the explicit description of polarisability on some crucial properties such as the resistance to amorphisation. This refined description of the interatomic interactions would certainly affect the description of the recrystallization process following a displacement cascade. In turn, the recovery phase would be better described using the core-shell model due to the lack of polarisability in the rigid-ion model.

\subsubsection{Classical molecular dynamics investigation of the formation and migration of dislocations}

In addition to the studies of point defects shown in the previous Sections, investigations of the extended defects created under irradiation in $\mathrm{UO}_{2}$ were also initiated. The structure of dislocations, an

Temperature (K)

(a)

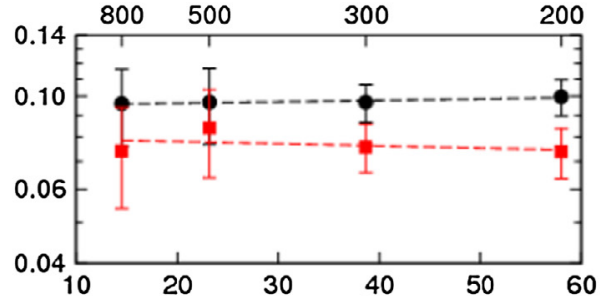

(c)

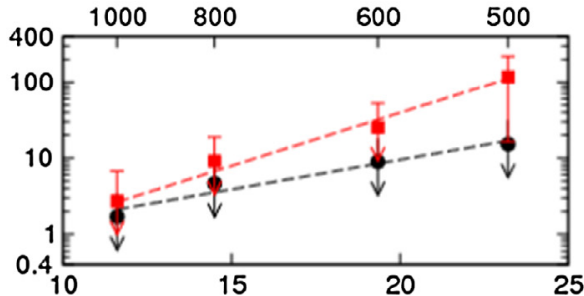

(e)

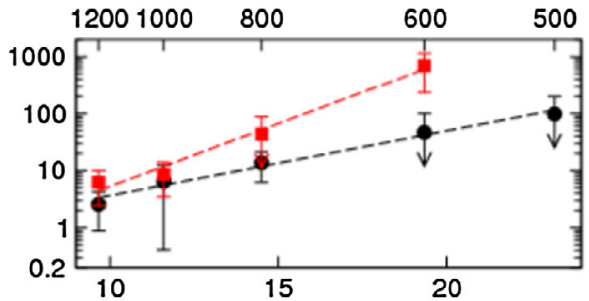

(b)

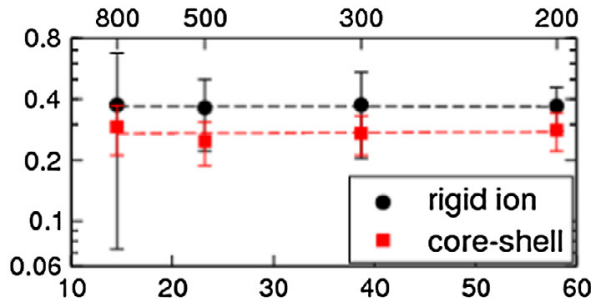

(d)

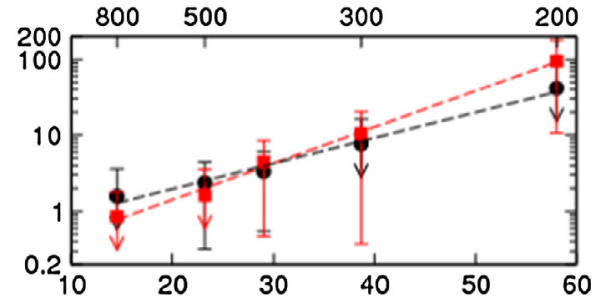

(f)

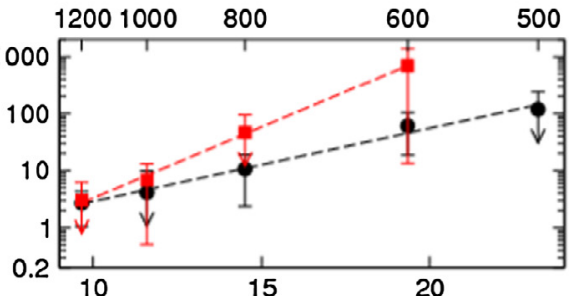

$1 / k_{B} T\left(e V^{-1}\right)$

Fig. 7. Arrhenius plots and best fits of the lifetimes of the oxygen FPs of rank (a) 1, (b) 2, (c) 3, (d) 4 of type I, (e) 4 of type II, and (f) 5 for different temperatures in the rigid-ion (black) and core-shell (red) model [61]. (For interpretation of the references to colour in this figure legend, the reader is referred to the web version of this article.) 
estimate of strain they induce on the surrounding lattice and the activation energy to dislocation creep were investigated using empirical pair potentials, principally the Morelon potential [62]. Pure edge and screw dislocations with $1 / 2[110]$ Burgers vectors were considered, these consisted of three edge dislocations with [1 10$]$, [001] and [1 112$]$ line directions respectively and a screw dislocation with a [1 10$]$ line direction. The simulations cells were created by rotating the fluorite $\mathrm{UO}_{2}$ lattice such that the dislocation line direction was parallel to the $z$-axis. The cell was periodic in the $z$-direction only and the line energy was determined by calculating the energy of ions contained within a cylindrical region, of radius $r$, centred on the dislocation and subtracting the energy of the same number of ions of perfect $\mathrm{UO}_{2}$ (i.e. $\sum N_{j} E_{j}$ ) lattice using:

$E(r)=E_{d}(r)-E_{p}(r) \cong E_{d}(r)-\sum_{j=U, O} N_{j}\left\langle E_{j}\right\rangle$

where, $E_{d}(r)$ is the energy of the cylinder containing the dislocation and $E_{p}(r)$ is the energy of a cylinder of the same radius containing pure $\mathrm{UO}_{2}$.

The calculated line energies for the four dislocations examined here are given in Fig. 8. This shows that the screw dislocation has the lowest line energy and that there is excellent agreement between the simulations (solid lines) and the predictions of linear elastic theory (dotted lines) beyond the dislocation core.

To quantify how easy it is to move a dislocation, the Peierl's barriers to slip in each of the three edge slip systems was calculated using the NEB method. This is defined as the minimum energy the atomic configuration must overcome to produce a second atomic configuration that is identical, apart from a displacement of the dislocation core by a single lattice spacing, as shown in Fig. 9.

The lowest energy barrier occurs for the $\{100\}\langle 110\rangle$ system $\left(2.5 \mathrm{eV} \mathrm{nm}^{-1}\right)$, while the $\{110\}\langle 110\rangle$ and $\{111\}\langle 110\rangle$ slip systems have Peierl's barriers of $4.6 \mathrm{eV} \mathrm{nm}^{-1}$ and $3.1 \mathrm{eV} \mathrm{nm}^{-1}$, respectively. The calculated values, showing the lowest Peierl's barrier on the $\{100\}$ slip plane, are in agreement with experiment for stoichiometric $\mathrm{UO}_{2}$ [63].

\subsubsection{Classical molecular dynamics simulations of collision cascades in} mono, bi and polycrystalline $\mathrm{UO}_{2}$

During reactor operation, the fission fragments generated within the oxide fuel lose their kinetic energy from two types of processes. At high energy, the fission fragment energy loss originates essentially in inelastic interactions, such as electronic excitations of atoms in the fuel matrix, which may result in the displacement of atoms located along the ion trajectory. At lower energies, energy loss occurs through ballistic collisions between

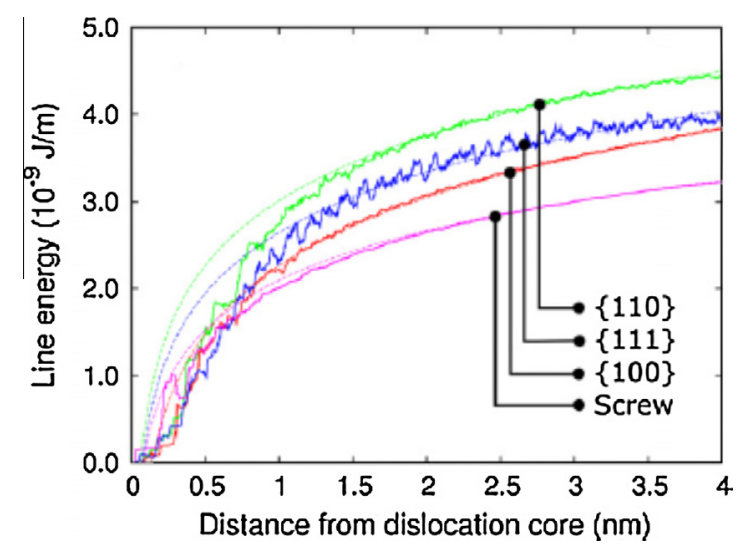

Fig. 8. Line energies of the dislocations as a function of the distance from the core [62]. Solid lines are those determined from the simulations and dotted lines are the calculated from linear elastic theory.

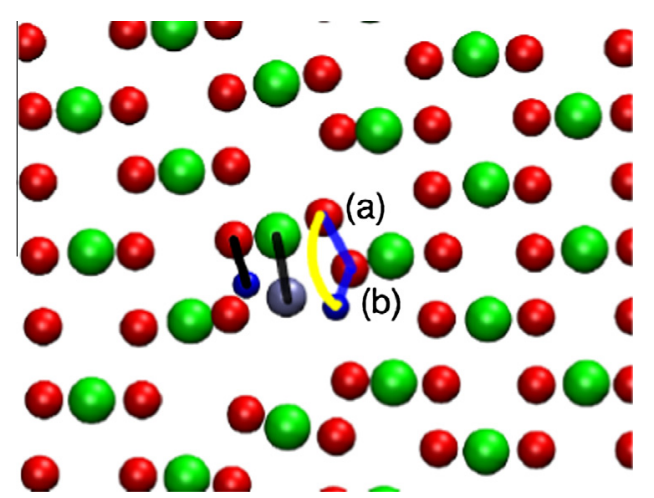

Fig. 9. Possible pathways for slip in $\mathrm{UO}_{2}$ on the $\{100\}\langle 110\rangle$ system [62]. Red and green spheres represent oxygen and uranium ions, respectively. Small blue spheres represent the position of the $\mathrm{O}-\mathrm{U}-\mathrm{O}$ triplet as the dislocation moves up the page. (For interpretation of the references to colour in this figure legend, the reader is referred to the web version of this article.)

atoms. The ballistic phase of radiation damage is often investigated using the simulation of displacement cascades starting from a primary knock-on atom (PKA) within the framework of molecular dynamics. In this method a single atom (the PKA) in the simulation cell is given a significant kinetic energy representing the ballistic interaction of radiation with the host matrix. This atom then collides with its neighbouring ions which are subsequently displaced and are able to displace other atoms. Different successive phases occur during a displacement cascade. First, within a few tenths of picoseconds, a ballistic phase is created during which long range migration of atoms can occur. This phase leads to the formation of sub-cascade branches. During a second stage, the energy of the cascade is thermally dissipated and a molten volume appears which lasts a few ps. Finally the temperature inside the box decreases progressively under the influence of the thermostat applied to atoms externally placed at the box boundaries since the system is completely relaxed after a few tens of ps.

There are a number of factors that can influence the evolution of radiation damage cascades in materials, in particular the choice of PKA (i.e. U or O), the amount of energy transferred to the PKA and the direction in which the PKA is initiated. The influence of the direction of the PKA was examined by performing simulations in which $0.4 \mathrm{keV}, 1 \mathrm{keV}$ and $10 \mathrm{keV}$ was assigned to the PKA in 24 different directions, while also taking care to examine the statistical effects associated with CMD simulations [64]. The total numbers of defects at the peak of radiation damage are plotted according to the direction in which the PKA was initiated in Fig. 10.

The results suggest that at relatively low PKA energies there is some anisotropy in the number of defects produced by the cascade. However, as the initiation energy is increased the direction in which the PKA is initiated becomes less significant.

The influence of grain boundaries on the primary damage state created by a recoil nucleus in the $\mathrm{UO}_{2}$ matrix was also investigated using classical molecular dynamics simulations [65]. This study was divided in two steps: first, the study of the structural properties of several symmetrical tilt boundaries for different misorientation angles ranging from $12.7^{\circ}$ to $61.9^{\circ}$, second the study of displacement cascades near these grain boundaries.

For all the grain boundaries studied, the structure around the interface up to about $10 \AA$ exhibits a perturbed fluorite lattice. However, in all cases, the structure after relaxation is stable. As indicated in Section 5.1.3 the type of defects present at the interface depends directly on the value of the misorientation angle. For the small angles, for instance for the $\Sigma 41$ grain boundary, the interface defects correspond to edge dislocations without any sig- 


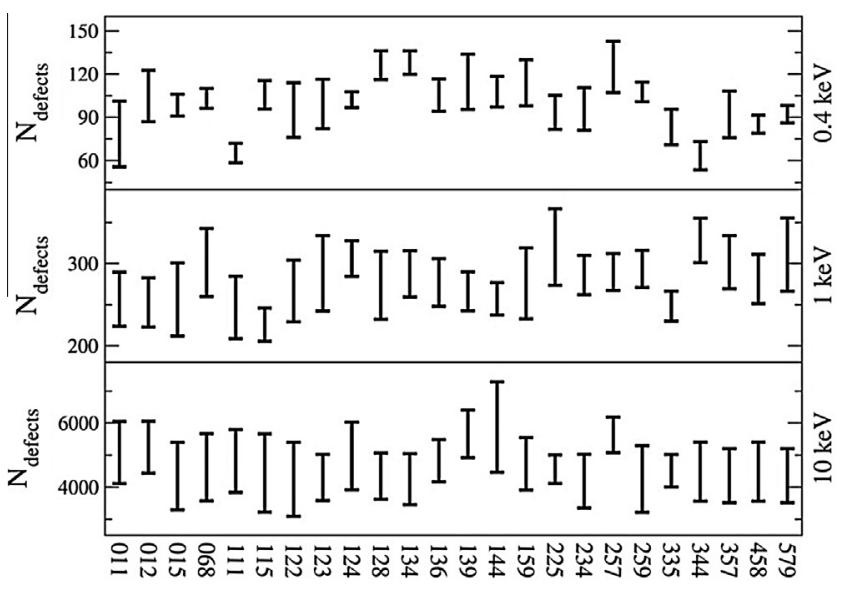

Fig. 10. Total number of defects at peak damage as a function of the crystallographic direction of the PKA [64]. The error bar represents the mean number of defects over the 10 simulations \pm one standard deviation.

nificant space between both mono-crystals. For misorientation angles greater than $20^{\circ}$, e.g. for $\Sigma 5$, there is a gap of about $1 \AA$ between the two mono-crystals which can be seen as a line of Schottky defects at the interface. Displacement cascades were initiated with a uranium projectile with a $10 \mathrm{keV}$ initial energy. In all cases, numerous point defects are created at the grain boundary region and the mobility of these defects is increased. However, cascade morphologies depend strongly on the structure of the grain boundaries (see Fig. 11).

Most of the displacements take place along the grain boundaries because of the large number of point defects at the GBs. Therefore, one can assume that displacements are enhanced by thermal diffusion and not due to ballistic collisions. Consequently, a large part of the PKA energy is dissipated along the GBs and few point defects are created in the grains.
In the case of poly-crystalline $\mathrm{UO}_{2}$, displacement cascades develop only in a few grains, and the grain size is not affected. This behaviour is similar to the one observed in bi-crystal symmetrical tilt boundaries with high misorientation angles. GBs diminish the number of collision sequences and act like sinks which trap the least energetic atoms. The role of the pre-existing defects at the interfaces is then of great importance for the creation of the primary damage due to a recoil nucleus. The grain size, however, probably plays an important role and needs to be considered in further studies.

5.1.7. Simulation of thermal spikes in mono and polycrystalline $\mathrm{UO}_{2}$ using classical molecular dynamics

In continuity with the work studying primary damage creation, thermal spikes were simulated in mono- and poly-crystalline $\mathrm{UO}_{2}$. While simulations of displacement cascades model the primary damage arising from collision sequences, which occurs for low energy projectiles, thermal spikes simulate the primary damage induced by high energy projectiles whose energy is mainly deposited in electronic excitations.

The thermal spikes simulated in monocrystalline $\mathrm{UO}_{2}$ all exhibit the same three stages [13]. The first stage of the thermal spike consists of the melting of the inner cylindrical zone, after less than $0.1 \mathrm{ps}$. Due to the difference in local density between the melted zone and the fluorite lattice, a shock-wave is rapidly emitted from this cylindrical zone. Subsequently a solidification phase occurs and lasts for approximately $50 \mathrm{ps}$. It is during this phase that residual damage appears. Nevertheless, no amorphisation of the fluorite matrix remains at the end of the thermal spike. Simulations performed in bulk $\mathrm{UO}_{2}$ show different types of damage according to the initial energies. Below a linear energy transfer equal to $16 \mathrm{keV} / \mathrm{nm}$, no defects or few point defects located within the thermal spike core are found.

For linear energy transfer greater than $16 \mathrm{keV} / \mathrm{nm}$ interstitial clusters appear in the $\{111\}$-plane leading to the formation of small edge dislocation loops with Burgers vector in the $\langle 110\rangle$
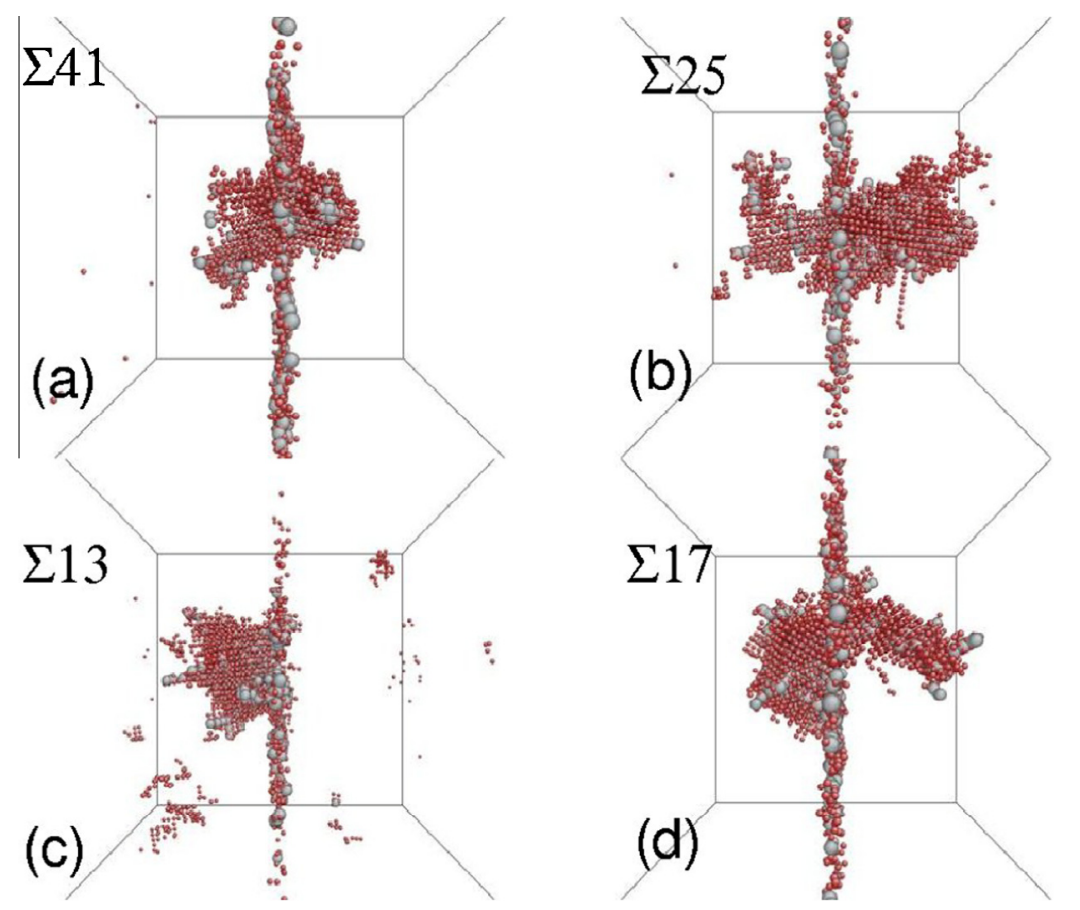

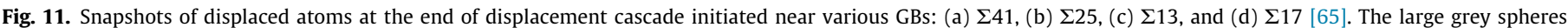

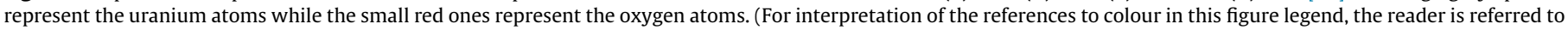
the web version of this article.) 


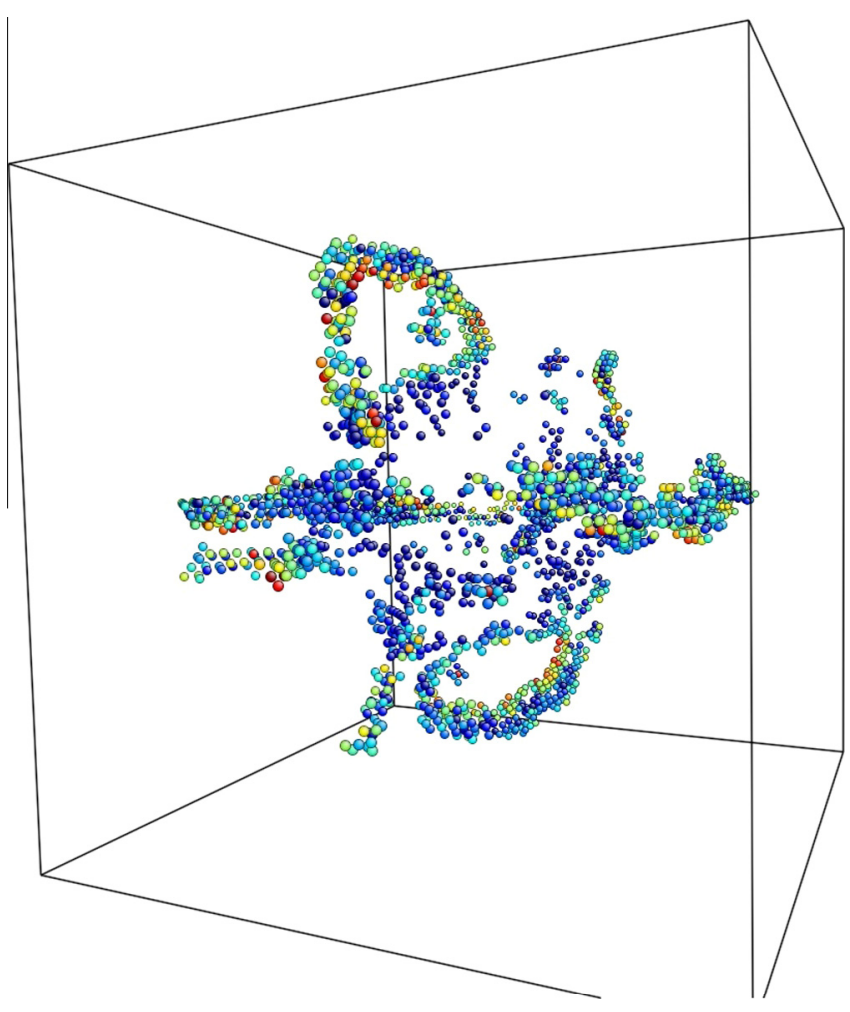

Fig. 12. Primary damage state after a thermal spike initiated with a linear energy transfer of $66 \mathrm{keV} / \mathrm{nm}$ [13]. Only the atoms with potential energies higher than in the bulk of the fluorite structure are shown. The colours indicate the potential energy leveled from blue for the lowest potential energies to red for the highest ones. Dislocation loops appear with burger's vector along the $\langle 110\rangle$ direction. (For interpretation of the references to colour in this figure legend, the reader is referred to the web version of this article.)

direction along the $\{111\}$ slip plane. For higher linear energy transfer energies $(>66 \mathrm{keV} / \mathrm{nm})$ nanometric edge dislocation loops are formed with Burgers vector in the $1 / 2\langle 110\rangle$ direction along the $\{100\}$ slip plane, as shown in Fig. 12. It is important to remark here that for the geometry considered, a linear energy transfer of $4 \mathrm{keV} /$ $\mathrm{nm}$ corresponds to a total energy dissipated in the simulation box equal to $80 \mathrm{keV}$. The damage created by thermal spikes is therefore significantly different from that created by displacement cascades.

Thermal spikes were then simulated in polycrystalline $\mathrm{UO}_{2}$. The polycrystal was created using the Voronoï polyhedral scheme. Therefore, each grain boundary has no particular structure and embeds several point defects. We have studied a simulation box of size $50 \times 50 \times 50 \mathrm{~nm}$ containing 60 grains of different fluorite orientations with minimum grain size of $3 \mathrm{~nm}$. A $66 \mathrm{keV} / \mathrm{nm}$ energy was chosen to initiate the thermal spike inside one grain since this initial energy created the maximum damage in the bulk (dislocation loops).
The results of the simulation are summarised in Fig. 13. Each image represents a snapshot of a $10 \AA$ slab during the thermal spike. Atoms are colour coded according to their potential energy: red atoms correspond to atoms in fluorite structure while other colours represent atoms in point defect or surrounded with point defects. This representation facilitates the localization and visualisation of grain boundaries.

After 4 ps a melting zone appears in the centre of the box (blue circle in the second snapshot). It is confined within one grain, which melts completely. This result is different to what was observed for displacement cascades in poly-crystal $\mathrm{UO}_{2}[65]$ where the melting zone crosses several grain boundaries because of subcascade branches. Here, the grain boundaries act as energy sinks. During the recrystallization phase, which follows the melting phase after about 28 ps, surrounding grains grow into the melted zone. At the end of the simulation only point defects are created. Unlike the simulation of a thermal spike carried out in single-crystal, no linear defects (dislocation loops) were created. These differences between mono- and poly-crystals show the importance of grain boundaries as energy sinks. However, grain size effect should play an important role and should be considered in further studies.

\subsubsection{Classical molecular dynamics study of radiation induced diffusion}

The contributions of the electronic and nuclear processes of energy loss during irradiation to the migration processes in the material are usually referred to as radiation induced diffusion (RID). Since this phenomenon is a key parameter of all fuel performance applications, it is essential to ascertain what physical processes contribute to it and to what extent. Classical molecular dynamics simulations using the interatomic potential developed by Morelon et al. [54] were performed in an attempt to estimate ballistic mixing effects and their possible temperature dependence [66]. Collision cascades were simulated at various temperatures from $300 \mathrm{~K}$ to $1400 \mathrm{~K}$, covering the temperatures relevant to normal operation and to long term storage conditions. Because of the computational resources required for such calculations, the energy of the projectile, a uranium primary knock-on atom (PKA), is limited to several tens of $\mathrm{keV}$. Simulations were therefore performed with various incident projectile energies (from $1 \mathrm{keV}$ to $80 \mathrm{keV}$ ) to enable the extrapolation of results to more realistic PKA energies.

Since a displacement cascade does not impact all the atoms present in the material, as thermal diffusion processes do, the mean square displacement of atoms has little meaning if given without the correspondent number of displaced atoms. The total square displacement $R_{X}^{2}$, i.e. the mean square displacement multiplied by the number of atoms displaced through the irradiation process, is here a more relevant parameter since the diffusion coefficient related to irradiation is directly connected to it. If the number of cascades $F$ which occur in a certain fuel volume during a given time
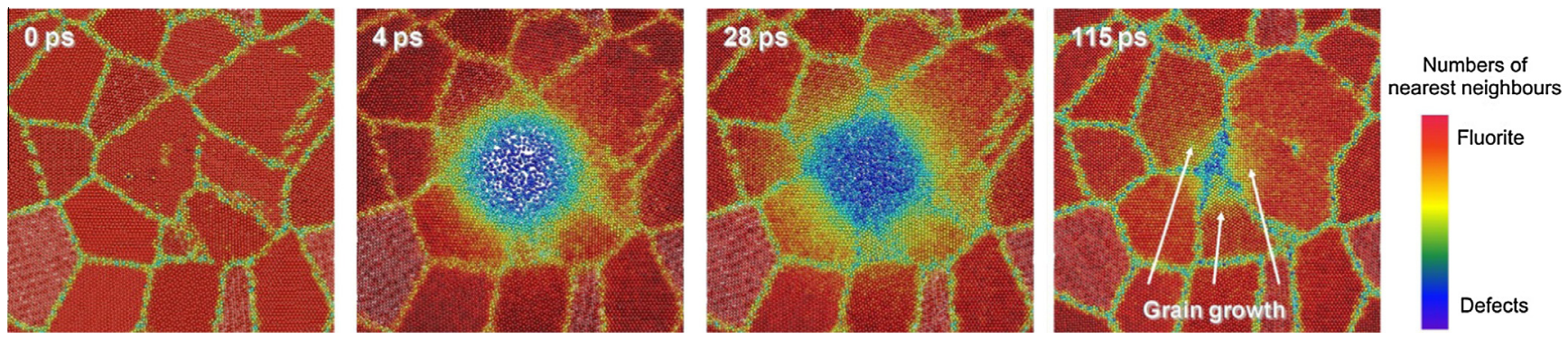

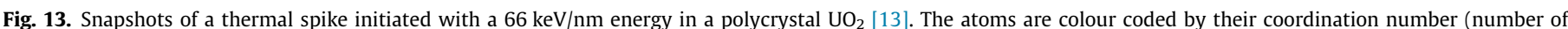

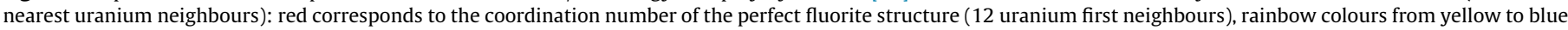

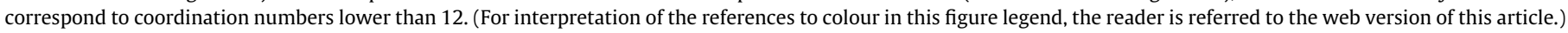


is known, it is then possible to estimate the diffusion coefficient $D_{n}(X)$ associated with the nuclear contribution to RID of element $X$ using Einstein's equation, as expressed below:

$D_{n}(X)=\frac{1}{6 \times V_{c} \times d_{X}} F \times V_{c} \times R_{X}^{2}=\frac{1}{6 \times d_{X}} F \cdot R_{X}^{2}=K . F$

where $d_{X}$ is the volume density of element $X, V_{c}$ the volume occupied by displaced atoms following a cascade event and $K$ the proportionality constant between the fission rate and the athermal diffusion coefficient. The $1 / 6$ factor comes from the symmetry of the uranium dioxide fluorite structure.

The results of the simulation show that the number of displaced atoms and the total square displacement are proportional to the PKA energy, which makes it possible to extrapolate them to higher energies. They also increase slightly with temperature. Then, as was shown previously [13], the total square displacement is approximately proportional to the number of cascade overlaps.

Fig. 14 shows the thermal diffusion and RID coefficients of oxygen and uranium in $\mathrm{UO}_{2}$ estimated from the current CMD results for normal operating (temperatures from 700 to $1400 \mathrm{~K}$, cascade density equal to $2 \times 10^{19} \mathrm{~m}^{-3} \mathrm{~s}^{-1}$ over 4 years) and long-term storage conditions (temperatures below $700 \mathrm{~K}$, cascade density less than $5 \times 10^{15} \mathrm{~m}^{-3} \mathrm{~s}^{-1}$ over 10,000 years) compared with the RID coefficients found in the literature (in some cases extrapolated).

It can be seen on Fig. 14 that radiation induced diffusion is an almost athermal process, as was expected at least in the nuclear energy loss regime. It can be seen on Fig. 14b that the calculated in-pile uranium diffusion coefficient describing the ballistic mixing lies two to three orders of magnitude below that determined experimentally under comparable conditions. It is therefore probable that nuclear collisions and the associated ballistic mixing do not provide an explanation for the observed movement of atoms under normal operating conditions. Electronic excitations which are not taken into account in the simulations and/or the enhanced diffusion of defects could explain this difference. For oxygen, experimental thermal diffusion coefficients range between $1 \times 10^{-20}$ and $1 \times 10^{-22} \mathrm{~m}^{2} \mathrm{~s}^{-1}$ at $700 \mathrm{~K}$ and are therefore comparable to the RID coefficients estimated here. At $1400 \mathrm{~K}$ on the other hand, the ballistic contribution to the diffusion coefficient seems negligible $\left(2 \times 10^{-22} \mathrm{~m}^{2} \mathrm{~s}^{-1}\right.$ in comparison with $1 \times 10^{-14}$ and $10^{-12} \mathrm{~m}^{2} \mathrm{~s}^{-1}$ at $\left.1400 \mathrm{~K}\right)$.

In long-term storage conditions, the values determined in this study are at least two to three orders of magnitude below those which were deemed relevant for spent nuclear fuels. The
Table 4

Incorporation energies of krypton and xenon atoms in $\mathrm{UO}_{2}$ calculated using the GGA and the GGA + $U$ approximation.

\begin{tabular}{llrlllll}
\hline Incorporation energies $(\mathrm{eV})$ & & Int. & $V_{U}$ & $V_{O}$ & $S_{1}$ & $S_{2}$ & $S_{3}$ \\
\hline $\mathrm{Kr}$ & DFT + U & 6.6 & 2.2 & 5.3 & 0.6 & 1.3 & 1.4 \\
$\mathrm{Xe}$ & DFT & 12.0 & 5.8 & 9.1 & $/$ & $/$ & $/$ \\
& DFT + U & 9.5 & 3.8 & 6.9 & 1.2 & 1.8 & 2.3 \\
\hline
\end{tabular}

phenomena mentioned above may be invoked to explain this difference.

These first results relative to the ballistic mixing under irradiation constitute a relevant dataset to feed models relative to the fuel behaviour under irradiation.

\subsection{Transport properties of fission gases in $\mathrm{UO}_{2}$}

\subsubsection{Electronic structure study of rare gas incorporation in $\mathrm{UO}_{2}$}

The incorporation of krypton and xenon was investigated in $\mathrm{UO}_{2}$ using DFT $+\mathrm{U}[43,8]$. The same calculation level and parameters was used as for the study of defect formation and migration (see Section 5.1.1). The impurities were introduced at various positions in the crystal lattice, in particular the oxygen and uranium substitution sites $\left(V_{O}\right.$ and $\left.V_{U}\right)$, the octahedral interstitial site (Int.) and in the three possible Schottky defects $\left(S_{1}, S_{2}\right.$ and $\left.S_{3}\right)$. The incorporation energies obtained are given in Table 4. Positive incorporation energies mean that the impurity is not favourably incorporated in the lattice.

It can be seen in Table 4 that the incorporation energies of $\mathrm{Kr}$ and $\mathrm{Xe}$ are all positive, which means that $\mathrm{Kr}$ and $\mathrm{Xe}$ are insoluble in $\mathrm{UO}_{2}$. These energies are high for all defects except for the Schottky defects (values around $1 \mathrm{eV}$ ). This suggests that if $\mathrm{Xe}$ and $\mathrm{Kr}$ are not soluble as interstitial or in $\mathrm{U}$ or $\mathrm{O}$ sites, they should be relatively easily incorporated in pre-existing trivacancies.

The incorporation energies calculated also show that $\mathrm{Kr}$ and $\mathrm{Xe}$ have a similar behaviour in $\mathrm{UO}_{2}$. Combined with the analysis of the atomic displacements (see Fig. 15) and charge redistribution observed after incorporation, the results obtained prove that steric effects are the main contribution to the incorporation of these rare gas atoms in $\mathrm{UO}_{2}$ as is expected because of their filled electron shell, as was also shown by Thompson and Wolverton [67] and Brillant et al. [68].
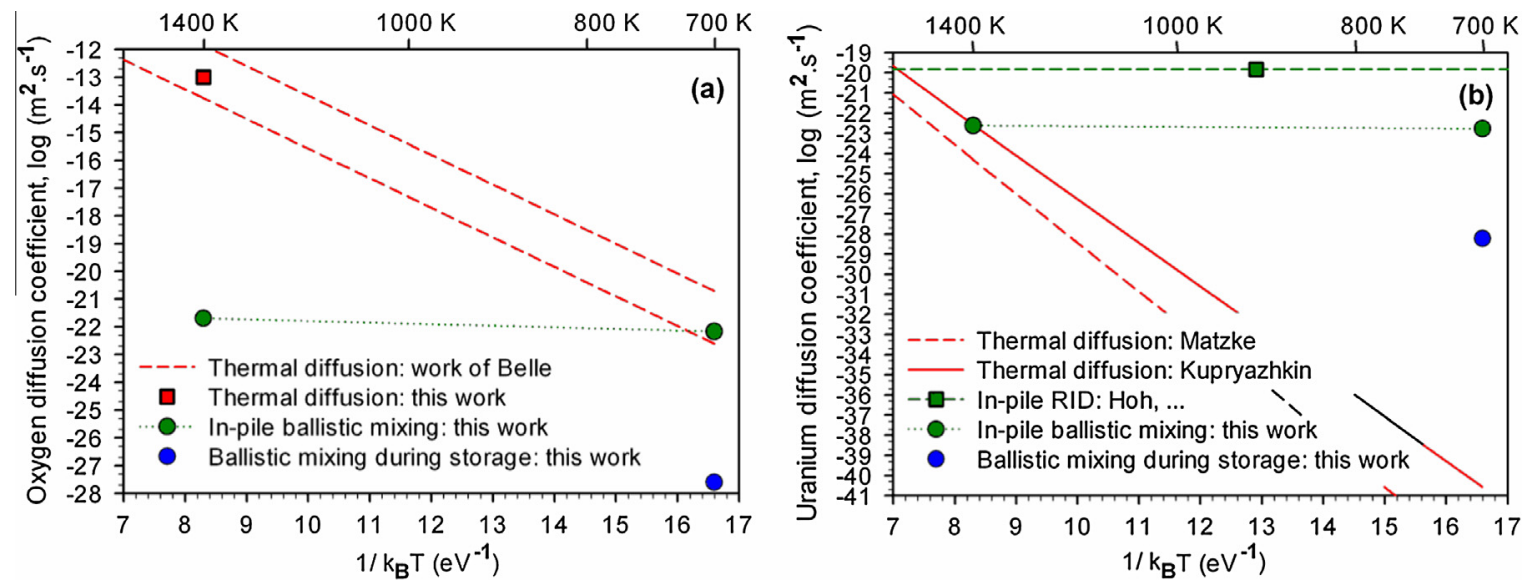

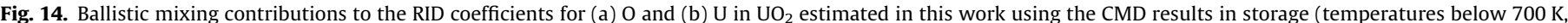

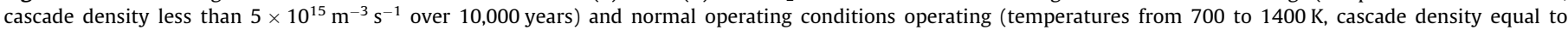
$2 \times 10^{19} \mathrm{~m}^{-3} \mathrm{~s}^{-1}$ over 4 years) compared to the thermal diffusion coefficients from the literature (references are listed in [66]). 


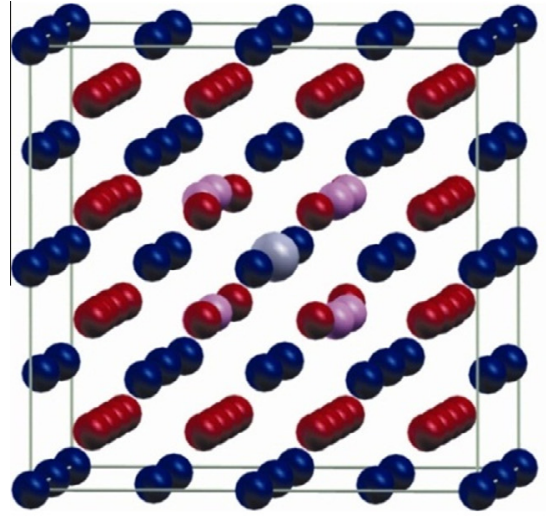

(a)

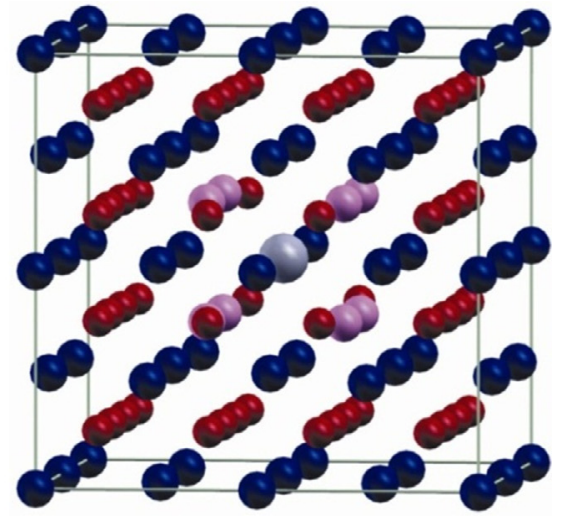

(b)

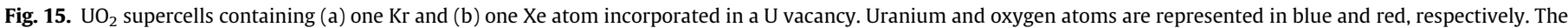

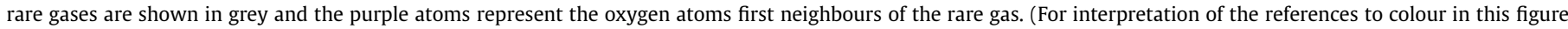
legend, the reader is referred to the web version of this article.)

\subsubsection{Empirical potential study of Xe incorporation in nanovoids}

The study of the incorporation of rare gases in larger defects is not yet possible using electronic structure methods and empirical potentials must then be used. The thermodynamics of the formation of Xe containing nanovoids and bubbles from point defects was investigated using an unusual disintegration procedure within the context of classical molecular dynamics simulations employing empirical pair potentials [69]. The Morelon empirical potential [54] was chosen for pure $\mathrm{UO}_{2}$ and a new potential developed during the F-BRIDGE project was used for $\mathrm{Xe}-\mathrm{U}$ and $\mathrm{Xe}-\mathrm{O}$ in $\mathrm{UO}_{2}$ [70]. This disintegration procedure allows the determination of the free energy of Xe atoms accommodated in the $\mathrm{UO}_{2}$ lattice and more importantly an estimation of the dynamic and temperature effects.

In the disintegration procedure the interactions with the target ion (i.e. the one for which the free energy is to be calculated) are reduced incrementally such that no entropy is created during each step and the process can be viewed as being reversible. The internal energy, $d U$, of the system can be calculated using:

$d U=d F+T \delta S$

where $d F$ is the change in the Helmholtz free energy, $T$ is the temperature and $\delta S$ is the change in entropy. As no entropy is created the total change in the entropy is simply that exchanged with the thermostat and Eq. (5) becomes:

$d U=d F+T \delta S=d F+\delta Q_{\text {reversible }}$

where $\delta Q$ is the variation in heat during a reversible reaction.

The variation in the internal energy during a reversible reaction may also be given by

$d U=\delta Q_{\text {reversible }}+\delta W_{\text {reversible }}$

where $\delta W_{\text {reversible }}$ is the work performed along the disintegration path, i.e. the free energy $\left(d F=\delta W_{\text {reversible }}\right)$.

The free energies of the Xe atoms incorporated at point defects in $\mathrm{UO}_{2}$ are presented in Table 5 . The data predicts that there is very little change in the free energy of Xe accommodated at point defects as the temperature increases. Xe sits preferentially in Schottky defects $(S)$, as was seen at $0 \mathrm{~K}$. At higher temperatures the oxygen sublattice starts to diffuse and so larger differences in the free energy are predicted due to the rearrangement of the oxygen ions surrounding the defect.

The free energies of each Xe atom in a series of nanovoids and bubbles with different radii and Xe densities were calculated using the disintegration procedure. The evolutions of the average Xe free
Table 5

Free energies of $\mathrm{Xe}$ (in $\mathrm{eV}$ ) accommodated at point defects as a function of temperature, compared to the energies obtained at $0 \mathrm{~K}$ using energy minimisation.

\begin{tabular}{llllll}
\hline Temperature $/ \mathrm{K}$ & $\mathrm{Xe}_{i}$ & $\mathrm{Xe}_{U}$ & $\mathrm{Xe}_{O}$ & $\mathrm{Xe}_{\text {dimer }}$ & $\mathrm{Xe}_{\text {Schottky }}$ \\
\hline $0[70]$ & 11.92 & 5.40 & 9.34 & 4.87 & 4.21 \\
300 & 11.71 & 5.45 & 9.27 & 4.87 & 4.23 \\
550 & 11.54 & 5.48 & 9.27 & 4.87 & 4.23 \\
800 & 11.07 & 5.56 & 9.22 & 4.89 & 4.23 \\
1050 & 10.81 & 5.56 & 9.14 & 4.82 & 4.24 \\
1300 & 10.73 & 5.64 & 8.98 & 4.77 & 4.64 \\
\hline
\end{tabular}

energy are presented as a function of the Xe:Schottky ratio and of the density of Xe in the bubble in Fig. 16.

Fig. 16a shows that as the Xe:Schottky ratio increases the average free energy of the Xe contained within the bubble increases until the Xe:Schottky ratio reaches approximately 1.6, after which the free energy remains relatively constant. The temperature also appears to have only a very small influence on the average free energy. By comparing the average free energies in the different bubble sizes at a Xe:Schottky ratio of 1 it is evident that there is a decrease in the average free energy of Xe going from a single $\mathrm{Xe}$ in a single Schottky defect to $4 \mathrm{Xe}$ atoms in the nanovoid and up to bubbles of 6 and $10 \AA$ Aadius, thus suggesting a driving force to bubble nucleation.

Besides, Fig. 16b shows that there is a significant difference between the free energy of Xe in a bubble in $\mathrm{UO}_{2}$ and pure Xe with the same density, and that this difference decreases as the size of the bubble increases. This difference is particularly apparent in the density region that has been observed experimentally as demarked by the vertical black lines in Fig. 16b. Therefore for the smallest bubbles in $\mathrm{UO}_{2}$ it is not appropriate to consider that the Xe gas in these bubbles will behave the same as the pure Xe case due to the $\mathrm{Xe} / \mathrm{UO}_{2}$ interface. For the $10 \AA$ radius bubbles the discrepancy is reduced but still significant. The equation of state of gaseous Xe EOS is therefore not valid for small bubbles.

\subsubsection{Empirical potential investigation of He migration in bulk $\mathrm{UO}_{2}$}

In addition to the incorporation of rare gases, their migration properties are important parameters of mesoscale models. The migration of $\mathrm{He}$ in $\mathrm{UO}_{2}$ was investigated $[71,72]$ using three sets of interatomic potentials: the Morelon et al. [54] and the Basak et al. [73] potentials, as well as the Yakub potential developed in the F-BRIDGE project [71]. 

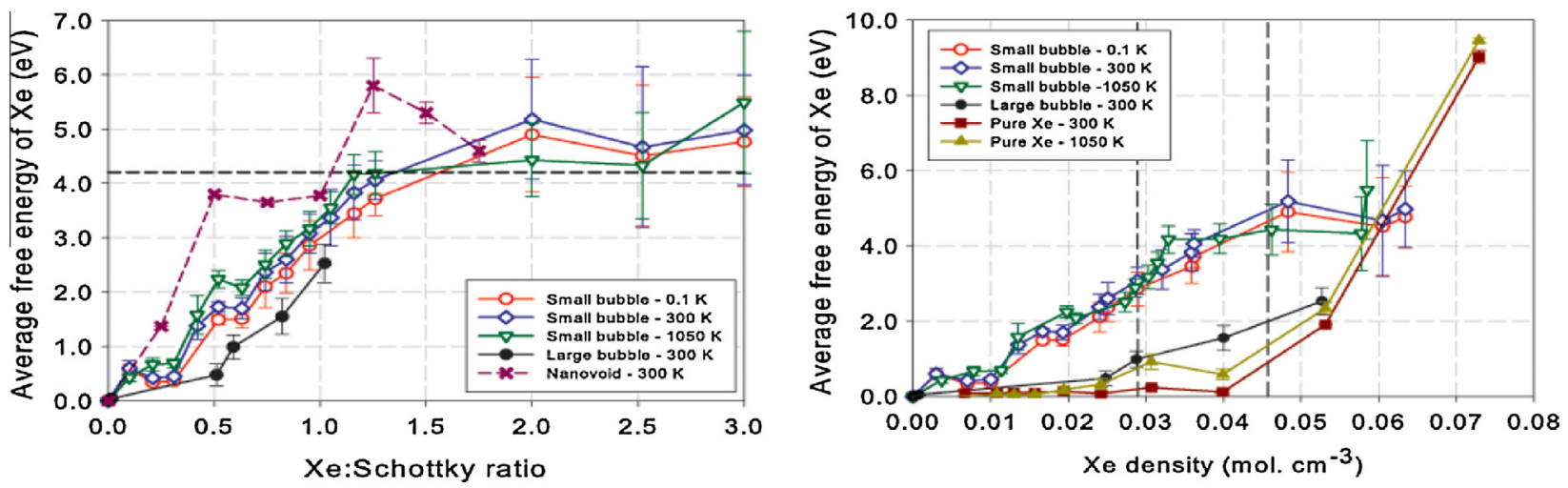

Fig. 16. Evolution of the average Xe free energy as a function (a) of the Xe:Schottky ratio and (b) of the density of Xe in the bubble [69].

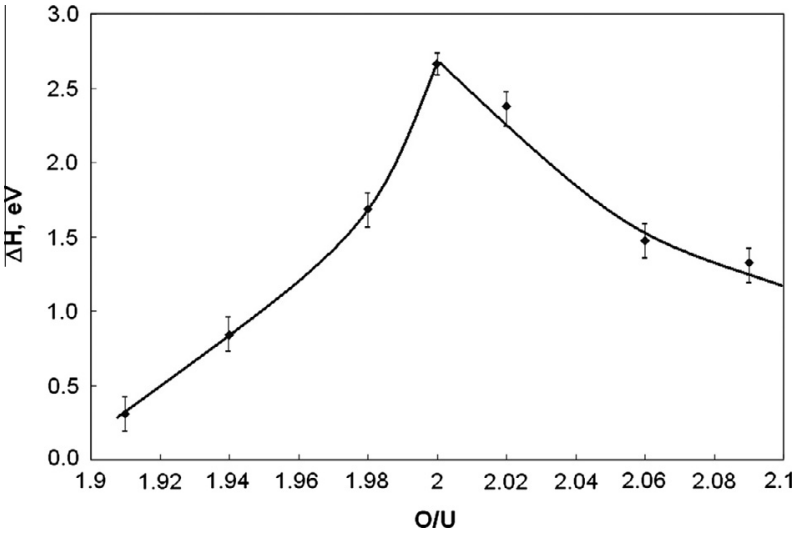

Fig. 17. Apparent activation energy of the helium diffusion in $\mathrm{UO}_{2+x}$ estimated using CMD with the free hopping approximation.

Since the diffusion of He is a slow process, a combination of conventional CMD and Monte Carlo (MC) methods was applied to accelerate the simulations using the Yakub potential. In a real oxide fuel, the solubility of He is extremely low and increases with temperature. The He concentrations in CMD simulations cannot be kept as low as in reality due to accuracy requirements and in order to maintain reasonable simulation times. CMD simulations were therefore performed at He concentrations higher than in the real dioxide, and the results were then extrapolated to lower concentrations. Fig. 17 shows the evolution of the apparent activation energy to He diffusion as a function of stoichiometry.

It can be seen on Fig. 17 that the apparent activation energy decreases when the deviation from stoichiometry increases. In hyperstoichiometric $\mathrm{UO}_{2}$ the activation energy is about $1.3 \mathrm{eV}$ for $\mathrm{O} / \mathrm{U}=2.09$. In the hypostoichiometric system it even becomes less than $0.5 \mathrm{eV}$ for $\mathrm{O} / \mathrm{U}=1.91$.

CMD simulations using the Basak and Morelon potentials were also performed at temperatures ranging from 1000 to $3000 \mathrm{~K}$. Various deviations from stoichiometry were considered in each case to derive an Arrhenius expression for He diffusion in the hyper- and hypostoichiometric domains. The Helium diffusion coefficients derived using the Morelon potential are plotted in Fig. 18.

The simulations suggest two migration mechanisms for helium. At low temperatures He diffusion is assisted by oxygen vacancies and exhibits a very low activation energy, around $0.5 \mathrm{eV}$. In the high temperature region, as well as for stoichiometric and hyperstoichiometric uranium dioxide, the activation energy presents a higher value, around $2.1 \mathrm{eV}$. Such behaviour was expected since at high temperature or in absence of structural defects, thermally generated defects dominate with concentrations showing a Boltzmann dependence on both the temperature and the defect formation energy. In that case, the activation energy is given by the sum of the migration energy and of the formation energy of the assisting defect.

The expression that can be derived for He intrinsic diffusion coefficient, i.e. in the stoichiometric and hyperstoichiometric domain, is as follows for the Morelon potential:
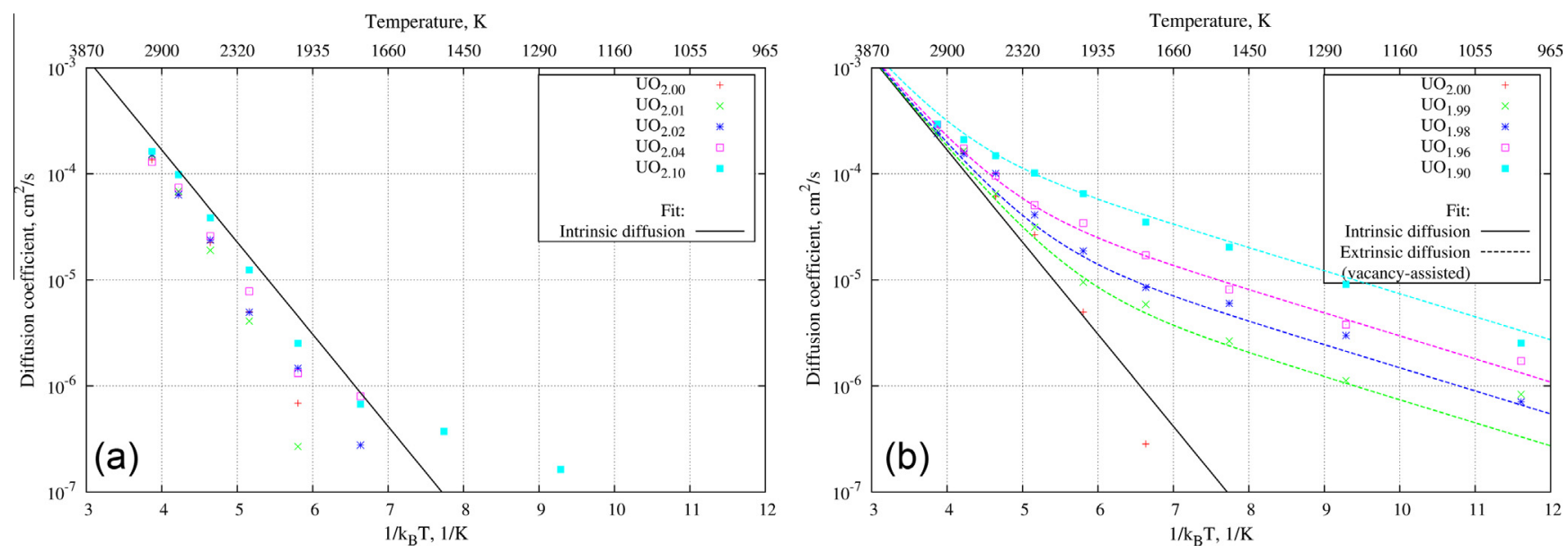

Fig. 18. He diffusion coefficient in the (a) hyperstoichiometric and (b) hypostoichiometric domains obtained using the Morelon potential [54]. 
$D_{\text {He,int }}=0.5 \exp \left(\frac{-2.0 \mathrm{eV}}{k_{B} T}\right) \mathrm{cm}^{2} / \mathrm{s}$

and for the $V_{O}$-assisted mechanism (hypostoichiometric domain),

$D_{\mathrm{He}, V_{O}}=0.011 \cdot|x| \cdot \exp \left(\frac{-0.5 \mathrm{eV}}{k_{B} T}\right) \mathrm{cm}^{2} / \mathrm{s}$

where $x$ is the (negative) deviation from stoichiometry.

NEB calculations were performed to elucidate the He migration pathways. He diffusion was considered both in a perfect lattice and in presence of one oxygen vacancy. NEB calculations confirmed the vacancy-assisted mechanism, which implies jumps between octahedral interstitial positions and oxygen vacancy locations, and showed that the observed migration energy corresponds to the barrier between the two sites. When there are no extrinsic oxygen vacancies (i.e. in the stoichiometric and hyperstoichiometric regimes) oxygen vacancies can still be created at elevated temperatures as intrinsic defects. It was found, however, that another diffusion mechanism, consisting of direct jumps of He atoms between octahedral sites with a migration energy around $2.2 \mathrm{eV}$, is operative at elevated temperatures. This is confirmed by both the MD and the NEB calculations. The migration energies calculated are reported in Table 6.

Finally, the effect of uranium vacancies on He diffusion was investigated. It was found that when He atoms are initially located in octahedral interstitial sites, no effect of uranium vacancies is observed on the diffusion coefficient. When He atoms are initially located at uranium vacancy sites, however, the diffusion coefficient decreases by 3 orders of magnitude. This shows the trapping effect of uranium vacancies on He atoms.

\subsubsection{Empirical potential investigation of Xe migration near grain boundaries or dislocations}

$\mathrm{Xe}$ is known to precipitate in nuclear fuel, either inside the grains (to form intragranular bubbles) or at grain boundaries (forming intergranular bubbles). The modelling of all steps leading to the formation of these bubbles is essential to properly describe the fission gas release process. The present section focuses on the mechanisms of diffusion leading to segregation or precipitation near grain boundaries and dislocation loops.

Following the investigation of Xe migration in bulk $\mathrm{UO}_{2}$ [74], the behaviour of Xe at grain boundaries was studied using classical molecular dynamics [75]. Two polycrystalline systems containing 6 grains with total sizes $10 \times 10 \times 10 \mathrm{~nm}$ (referred to as small system) and $20 \times 20 \times 20 \mathrm{~nm}$ (called large system) were constructed. The simulations performed enabled the calculation of the diffusion coefficient at grain boundaries as a function of temperature which is shown in Fig. 19.

The fit of the data of Fig. 19 yields activation energies to the Xe migration in the bulk and the grain boundaries equal to 2.4 and $0.9 \mathrm{eV}$, respectively. A faster diffusion coupled to a lower activation energy is observed at the grain boundaries compared to the bulk, as was seen for $U$ (see Section 5.1.3). Uranium and xenon atoms show similar activation energies, in agreement with the hypothesis that Xe migration is assisted by uranium defects. It should be noted that Xe clustering occurs at grain boundaries.

\section{Table 6}

He migration energies (in eV) between two octahedral interstitial positions (OIS) and between an interstitial position and an oxygen vacancy, NEB calculations with Basak and Morelon potentials.

\begin{tabular}{lll}
\hline Migration mechanism & Basak & Morelon \\
\hline $\mathrm{He}_{\mathrm{OIS}} \Rightarrow \mathrm{He}_{\mathrm{OIS}}$ & 3.5 & 2.26 \\
$\mathrm{He}_{\mathrm{OIS}} \Rightarrow \mathrm{He}_{\mathrm{Vo}}$ & 0.42 & $0.42-0.48$ \\
\hline
\end{tabular}

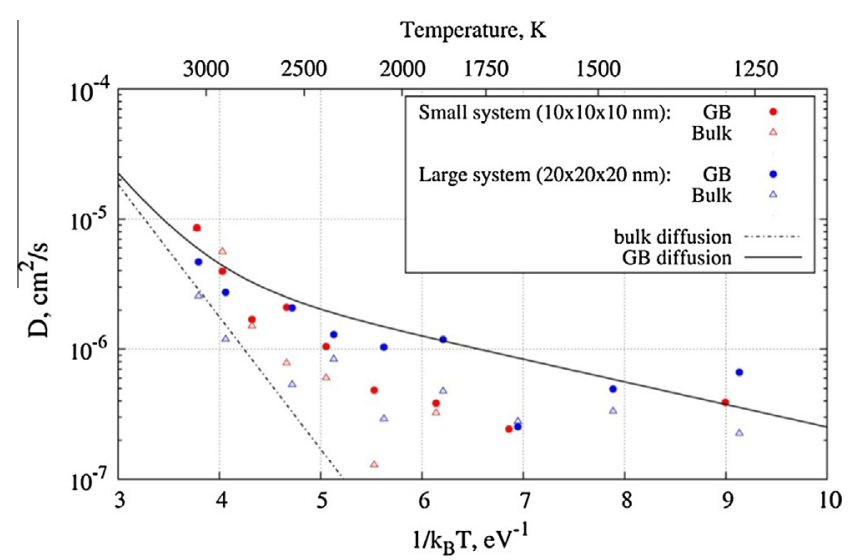

Fig. 19. Diffusion coefficient of xenon atoms as a function of temperature. Atoms located at grain boundaries are in red and purple; bulk atoms in blue and light blue. (For interpretation of the references to colour in this figure legend, the reader is referred to the web version of this article.)

The effect of microstructure on segregation characteristics of Xe was then explored [76] by substituting uranium ions by Xe in the previously determined atomic structures of dislocations: edge and screw, both with Burgers vectors of $\langle 110\rangle$ (see Section 5.1.5). The segregation energy is thus the difference between the energy of $\mathrm{Xe}$ in a $\mathrm{U}$ vacancy in the bulk and near the dislocation. Fig. 20 shows plots of the Xe segregation energy as a function of the distance from the dislocation, as well as a contour plot of the energies in the plane perpendicular to the dislocation direction. For both dislocation types the Xe energy increases as the distance from the dislocation core increases. The segregation energies for the edge and screw dislocations were predicted to be approximately $2.7 \mathrm{eV}$ and $5.5 \mathrm{eV}$, respectively. Xe segregation to dislocations is therefore found to be thermodynamically favourable and more favourable to a screw dislocation than to an edge dislocation by approximately $3 \mathrm{eV}$.

For the edge dislocation the lowest energy segregation site is in the tensile region of the strain field close to the dislocation core. This result is intuitive as the $\mathrm{Xe}_{U}$ defect is predicted to have a positive defect volume. Interestingly, despite this positive defect volume the segregation of $\mathrm{Xe}$ into the compressive region of the dislocation is preferable to bulk $\mathrm{UO}_{2}$. The screw dislocation, on the contrary, does not possess a significant region of hydrostatic strain and as a result the perturbation caused by the incorporation of Xe is significantly less than for the edge dislocation.

In order to provide context we compare these segregation energies obtained for dislocations to those obtained for the $\Sigma 5$ (tilt), $\Sigma 5$ (twist) and random grain boundaries discussed in reference [76]. Segregation energies for the $\Sigma 5$ (tilt), $\Sigma 5$ (twist) and random grain boundaries were found to be $4.1 \mathrm{eV}, 1.0 \mathrm{eV}$ and $6.4 \mathrm{eV}$, respectively. Therefore, the most thermodynamically favourable site for Xe segregation is at a randomly arranged grain boundary followed by accommodation near the core of a screw dislocation.

\subsubsection{CMD investigation of rare gas resolution under irradiation}

Bubbles act as traps for noble gases in nuclear fuels. However, this trapping effect is only temporary because of interaction of these bubbles with energetic particles that lead to partial resolution or even full destruction of the bubble. This process, generally referred to as irradiation-induced resolution, has been known for more than 40 years. A first interpretation is the Nelson model [77] where direct elastic collision with fission fragments or energetic PKA results in the ejection of atoms, one by one, from the bubble. That mechanism has later been called homogeneous resolution. Com- 

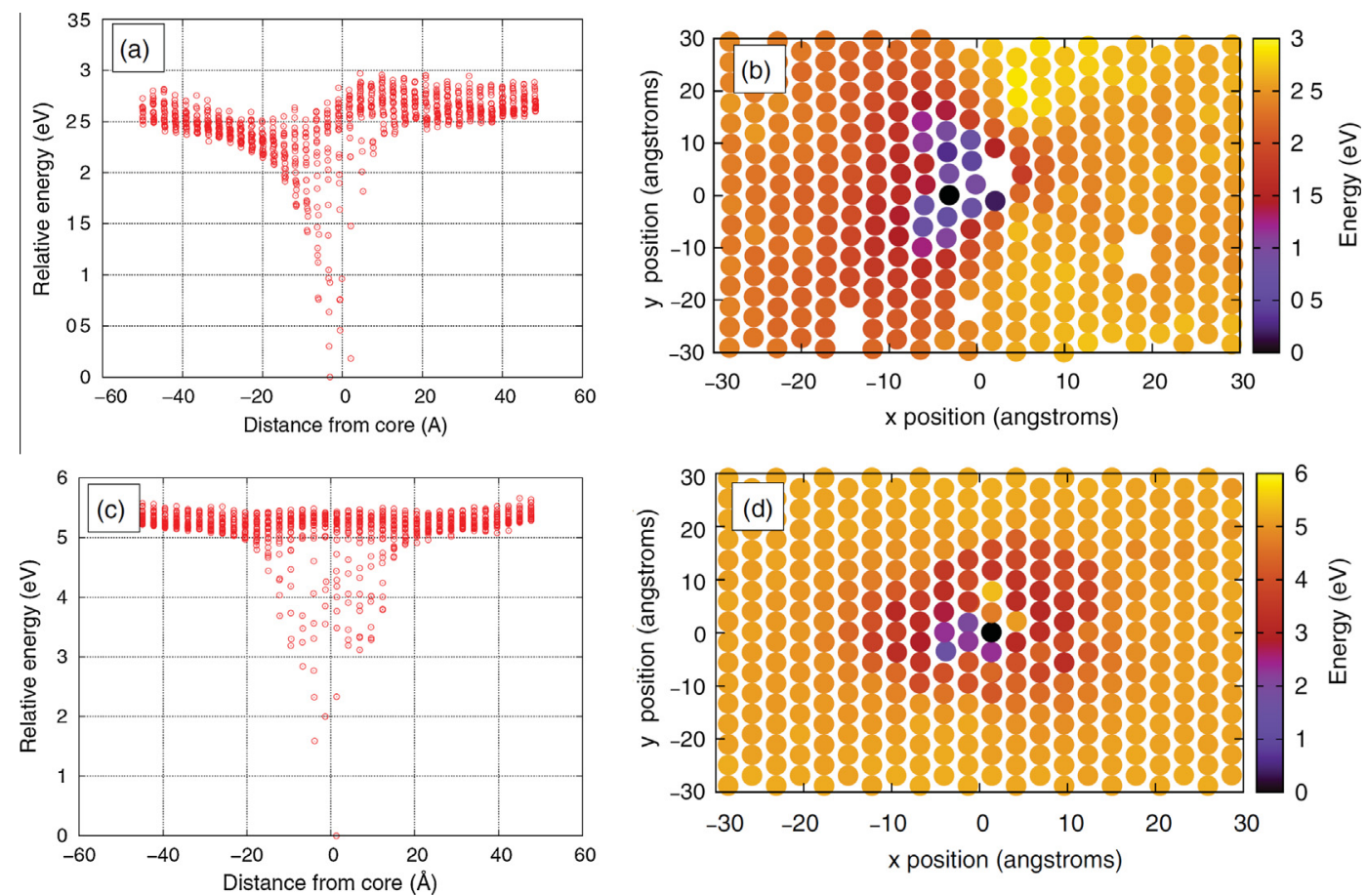

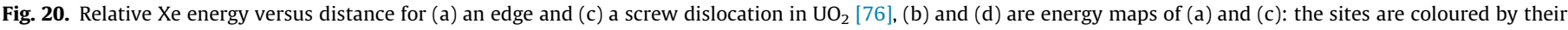

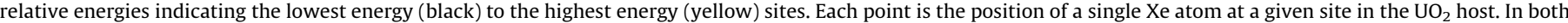

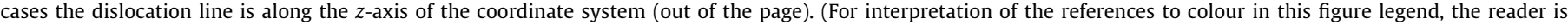
referred to the web version of this article.)

mensurate with the work of Nelson, Turnbull and Cornell [78] proposed another model by which the energy lost by fission fragments through electronic excitation favours a "sputtering" mechanism where blocks of material $(1.0-2.0 \mathrm{~nm}$ size) traverse the bubble, leading to gas resolution. This mechanism has later been called heterogeneous resolution. In the initial model of Turnbull, bubbles are assumed to be partially or totally destroyed when they are traversed by a fission fragment. The proposed resolution rate is proportional to the bubble content, with an estimation of 200 resolved atoms per traversing fission fragment for a $10 \mathrm{~nm}$ bubble. Later on, the model was adapted to account for an interaction distance between the fission fragment path and the bubble (the track radius), which would, de facto, lower the estimated number of resolved atoms per fission fragment.

The efficiency of both types of resolution processes was investigated using molecular dynamics techniques. Elastic collisions that were proposed by Nelson to explain gas resolution operate mostly below a few hundred keV for high energy ions and lead to a ballistic process similar to that observed during the recoil of an atom after neutron scattering or radiative emission such as $\alpha$-decay. Such collision cascades have been studied using the PKA approach, where the initial collision is modelled through an impact energy given to a regular lattice atom (cf. Section 5.1.6). This approach was applied to helium $[79,80]$ and xenon bubbles [81] in $\mathrm{UO}_{2}$ to illustrate the resolution from small intragranular bubbles.

It was found that the interaction of helium bubbles with uranium PKAs with energies between 10 and $50 \mathrm{keV}$ resulted in gas resolution via two mechanisms. First, energetic recoil during the ballistic phase and second via leakage into the highly defective region of $\mathrm{UO}_{2}$ at the bubble surface: damage assisted resolution. Examples of these mechanisms can be viewed in Fig. 21. Helium bubbles of increasing density were investigated and resolution was observed in all cases both by damage assisted and ballistic mechanisms. The probable mechanism of resolution (i.e. damage assisted or ballistic) was determined by monitoring of the resolved atoms' kinetic energy throughout the simulation. The amount of total resolution was found to increase with increasing bubble density. It is unclear, however, if the proportion of atoms resolved by the two mechanisms also varies as a function of bubble pressure.

In the case of $\mathrm{Xe}$, displacement cascades simulations initiated by PKA with energies between 10 and $50 \mathrm{keV}$ were performed in the presence of low, medium and high density xenon bubbles of radius $2.0 \AA$ and $2.5 \mathrm{~nm}$. No resolution was observed in the majority of simulations (68\%). The maximum resolution, which occurred only in two simulations, was 3 atoms emitted from the bubble via a ballistic mechanism. There was no significant increase in the average kinetic energy of the gas atoms in the bubble nor was leakage observed into an amorphous region, as seen with helium.

The investigations also covered the damage created by thermal spikes (cf. Section 5.1.7) since Turnbull's model is based on the fact that the major contribution to the slowing down of swift ions actually originates from interactions with electrons. The dependence of resolution with bubble size, bubble pressure, thermal spike radius and energy deposited by the thermal spike was investigated. The numbers of resolved atoms for various conditions are illustrated in Fig. 22.

At low energy deposition rate in the lattice, no resolution was observed. Resolution was only observed starting from an energy transfer equal to $13 \mathrm{keV} / \mathrm{nm}$. The series of simulations performed indicate that the number of resolved atoms increases almost linearly (over the range covered in our simulations) with the thermal spike energy as follows:

$N_{\text {at.resol }}=11.03\left(E_{\mathrm{av} / \mathrm{at}}-3.17\right)$

where $N_{\text {at.resol }}$ is the number of resolved atoms and $E_{\text {av/at }}$ is the average initial energy (in $\mathrm{eV}$ ) per atom deposited by the thermal spike. Interestingly, no dependence of this quantity with the bubble size or with the number of gas atoms they initially contain was observed.

These results show that displacement cascades and thermal spikes exhibiting similar energy transfers to the lattice give rise to very different resolution. Displacement cascades result in limit- 


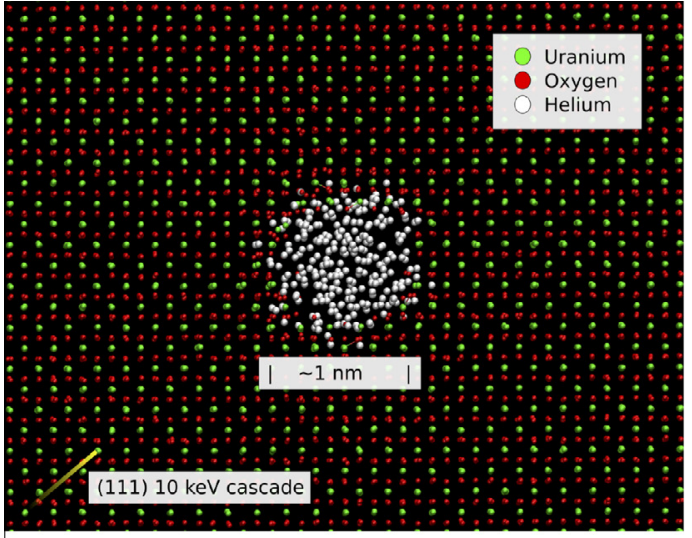

a

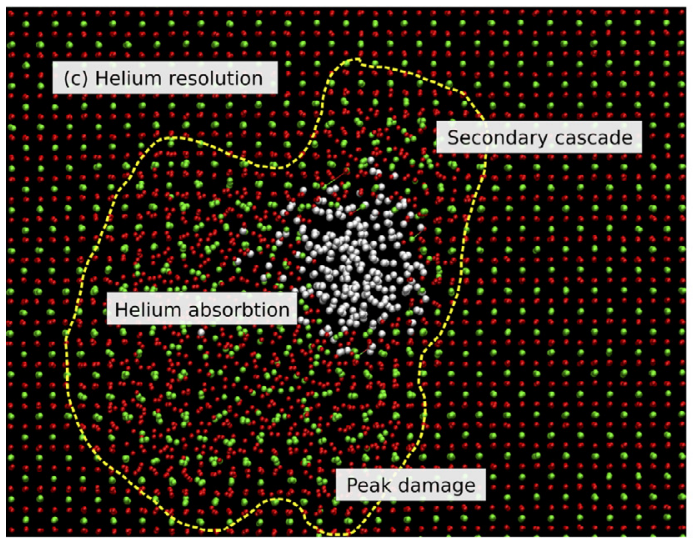

C

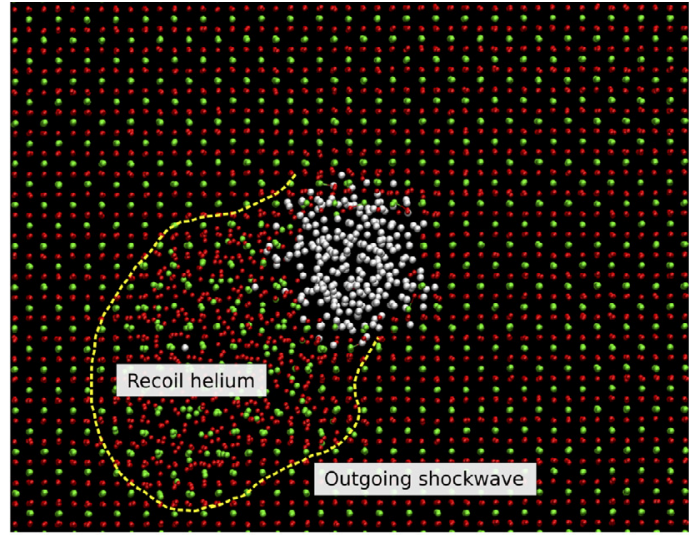

b

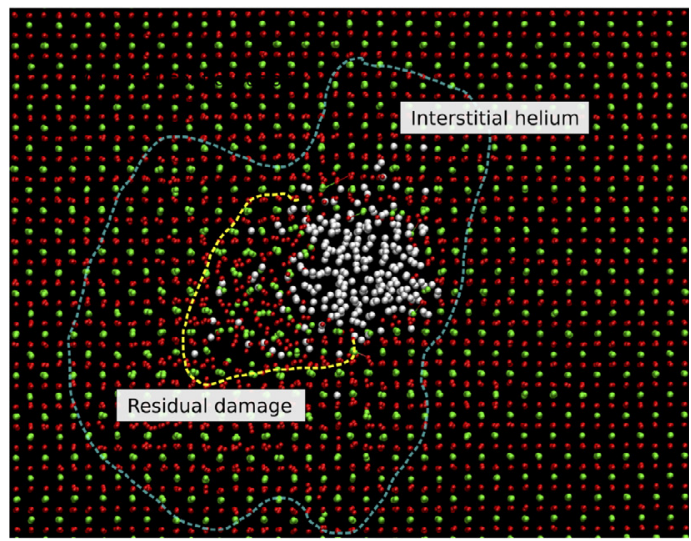

d

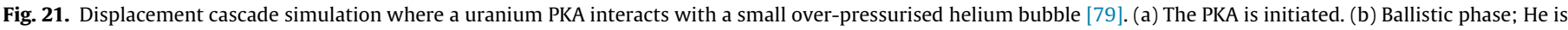

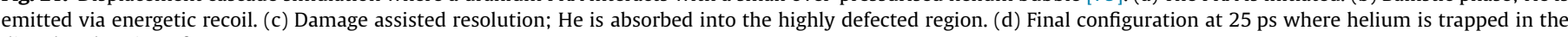
disordered region of $\mathrm{UO}_{2}$.

ed Xe resolution: a maximum of three atoms were resolved by a $50 \mathrm{keV}$ PKA during the initial ballistic phase (first tenth of picoseconds). Much larger resolution is observed for thermal spikes where the number of resolved atoms increases linearly with the deposited energy starting from a threshold value of approximately $13 \mathrm{keV} /$

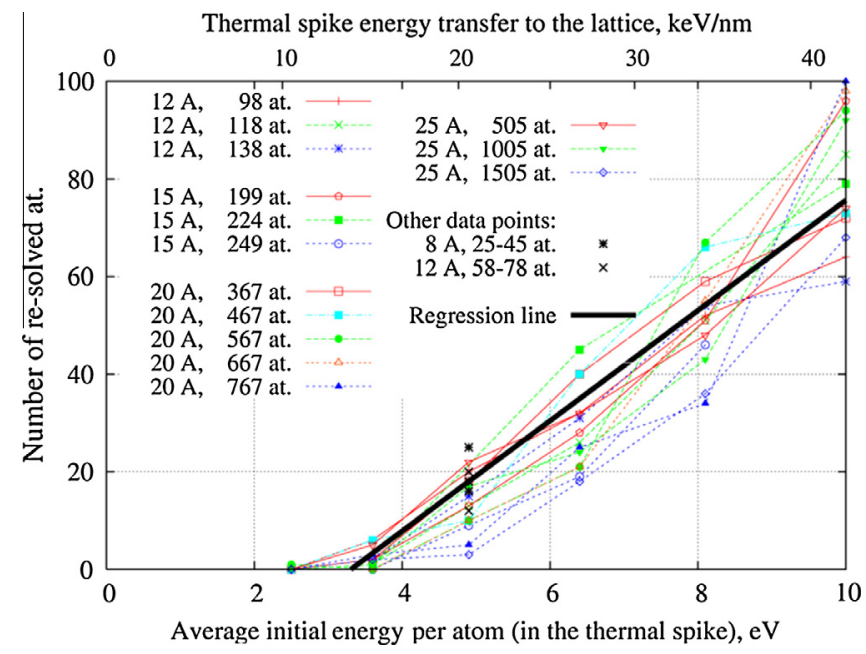

Fig. 22. Number of resolved Xe atoms as a function of the thermal spike energy, plotted for various bubble sizes and gas density in the bubble [81]. $\mathrm{nm}$. Resolution by thermal spike occurs during the whole duration of the event (melting and cooling down phases).

Atomic scale simulations allowed us to assess the mechanisms involved in earlier descriptions of resolution models (Nelson and Turnbull models). Although this work supports the "bubble destruction" approach of Turnbull, a major outcome is that bubble size and bubble density do not influence the resolution parameter, at least over the range covered in the study. The upper limit of number of atoms resolved which is independent from bubble size or pressure implies that from a certain size, bubbles can no longer be fully destroyed, leading to an increase of bubble size due to trapping. Uncertainties remain, specifically on the proportion of the electronic stopping power that is actually converted to lattice energy but our estimation is that at the highest stopping power that could be expected from a fission fragment, the number of resolved atoms per bubble would be between 5 and 20, the latter value being obtained if one assumes full conversion.

\section{Multiscale approach: summary of the links between the atomic and mesoscopic scales}

This section synthesises the results obtained in the development of the multiscale approach by showing the parameters of mesoscale models needed for the description of the atomic transport properties of defects and rare gases in $\mathrm{UO}_{2}$ which were calculated using atomic scale methods during the F-BRIDGE project and those which remain to be determined. 
Table 7

Input parameters of mesoscale parameters concerning the thermal evolution of $\mathrm{UO}_{2}$ calculated using atomic scale methods during the F-BRIDGE project and those which must still be determined.

\begin{tabular}{|c|c|c|}
\hline Mesoscale parameters & Electronic structure & Empirical potentials \\
\hline $\begin{array}{l}\text { Point defect formation } \\
\text { energies: } \mathrm{Vu}, \mathrm{Vo} \text {, } \\
\text { Vuo, } \mathrm{VuO}_{2}, \mathrm{Io}, \mathrm{Io}_{2} \text {, } \\
\mathrm{Iu}, \mathrm{FP}\end{array}$ & $\begin{array}{l}\text { Calculated using GGA } \\
\text { and GGA + U (see } \\
\text { Section 5.1.1) }\end{array}$ & $\begin{array}{l}\text { Rigid ion and core-shell } \\
\text { (CS) pair potentials (see } \\
{[36] \text { ) }}\end{array}$ \\
\hline \multirow[t]{2}{*}{$\begin{array}{l}\text { Migration energies of } \\
\text { vacancies and } \\
\text { interstitials in bulk }\end{array}$} & $\begin{array}{l}\text { Static calculations for Vu, } \\
\text { Vo, Io and Iu using GGA } \\
\text { and GGA + U (see } \\
\text { Section 5.1.1) }\end{array}$ & $\begin{array}{l}\text { Static and dynamical } \\
\text { calculations for } \mathrm{Vu}, \mathrm{Vo} \text {, Io } \\
\text { and Iu using rigid ion and } \\
\text { core shell potentials (see } \\
{[36] \text { ) }}\end{array}$ \\
\hline & $\begin{array}{l}\text { Calculations must be } \\
\text { done for Vuo, } \mathrm{Vuo}_{2} \text {, Io, } \\
\mathrm{Io}_{2}\end{array}$ & $\begin{array}{l}\text { Calculations must be } \\
\text { done for Vuo, } \mathrm{VuO}_{2} \text {, Io, } \\
\mathrm{Io}_{2}\end{array}$ \\
\hline $\begin{array}{l}\text { Migration energies near } \\
\text { extended defects } \\
\text { (vacancy clusters, } \\
\text { dislocations) or grain } \\
\text { boundary }\end{array}$ & $\begin{array}{l}\text { Calculations of model } \\
\text { systems could be done }\end{array}$ & $\begin{array}{l}\text { Calculations near } \\
\text { dislocations and grain } \\
\text { boundaries using rigid } \\
\text { ion potentials (see } \\
\text { Section } 5.1 .3 \text { ) }\end{array}$ \\
\hline $\begin{array}{l}\text { Formation/bonding } \\
\text { energies of extended } \\
\text { defects }\end{array}$ & $\begin{array}{l}\text { Very small clusters could } \\
\text { be done }\end{array}$ & $\begin{array}{l}\text { Dislocations studied } \\
\text { using rigid ion potentials } \\
\text { (see Section } 5.1 .5 \text { ) }\end{array}$ \\
\hline $\begin{array}{l}\text { Migration energies of } \\
\text { extended defects }\end{array}$ & $\begin{array}{l}\text { Currently very difficult } \\
\text { because of the } \\
\text { computational cost }\end{array}$ & $\begin{array}{l}\text { Dislocations studied } \\
\text { using rigid ion potentials } \\
\text { (see Section } 5.1 .5 \text { ) }\end{array}$ \\
\hline $\begin{array}{l}\text { Incorporation and } \\
\text { migration energies of } \\
\text { isolated fission gas } \\
\text { and helium in bulk }\end{array}$ & $\begin{array}{l}\text { Incorporation studied in } \\
\text { GGA + U (see } \\
\text { Section } 5.2 .1 \text { ) migration } \\
\text { remains to be done }\end{array}$ & $\begin{array}{l}\text { Static calculations using } \\
\text { rigid ion and core-shell } \\
\text { potentials (see } \\
\text { Section } 5.2 .3 \text { ) }\end{array}$ \\
\hline $\begin{array}{l}\text { Migration energies of } \\
\text { isolated fission gas or } \\
\text { helium in or near } \\
\text { grain boundary or } \\
\text { extended defects }\end{array}$ & $\begin{array}{l}\text { Calculations of model } \\
\text { systems could be done }\end{array}$ & $\begin{array}{l}\text { Static calculations using } \\
\text { rigid ion potentials (see } \\
\text { Section } 5.2 .4 \text { ) }\end{array}$ \\
\hline $\begin{array}{l}\text { Energy of rare gas in } \\
\text { bubbles/equation of } \\
\text { state }\end{array}$ & $\begin{array}{l}\text { Currently very difficult } \\
\text { because of the } \\
\text { computational cost }\end{array}$ & $\begin{array}{l}\text { Calculation for Xe using } \\
\text { rigid ion potential (see } \\
\text { Section } 5.2 .2 \text { ) }\end{array}$ \\
\hline
\end{tabular}

\subsection{Thermal evolution}

Table 7 summarises the status of the calculations of the parameters concerning the thermal evolution of $\mathrm{UO}_{2}$ using atomic scale methods. The parameters in orange are data that can be presently calculated but could not be obtained by the end of the F-BRIDGE project. The red colour indicates parameters that are still very difficult to obtain.

It can be seen in Table 7 that significant progress was made and that most parameters concerning the thermal evolution of $\mathrm{UO}_{2}$ were calculated using state of the art empirical potential or electronic structure methods. These parameters were used in the mesoscopic models developed in the project and presented in Section 2. Of course, because of the complexity of the systems and of the processes investigated, the results shown here will have to be refined and extended in the future.

The high computational cost of electronic structure calculations, in particular for actinide compounds, induces very strict limitations on the size of systems that can be considered, as well as on the time of dynamic calculations. The investigations therefore focus on point defects and on the static investigation of migration pathways. Empirical potential studies complement the electronic structure investigations by enabling much more extensive dynamical investigation and the study of larger systems, including grain boundaries and extended defects. The common investigation of the properties of point defects enables the validation of the empirical potential results by comparison with the electronic structure results.
Table 8

Input parameters of mesoscale parameters concerning irradiation processes calculated using atomic scale methods during the F-BRIDGE project.

\begin{tabular}{ll}
\hline Mesoscale parameters & Empirical potentials calculations \\
\hline Damage created & $\begin{array}{l}\text { Number and types of defects created by } \\
\text { "small" displacement cascades using rigid ion } \\
\text { and shell model pair potentials (see } \\
\text { Section 5.1.6), as well as by thermal spikes } \\
\text { using rigid ion pair potentials (see } \\
\text { Section 5.1.7) }\end{array}$ \\
\hline Resolution of defects & $\begin{array}{l}\text { Recombination of O Frenkel pairs using rigid } \\
\text { ion and shell model pair potentials (see }\end{array}$ \\
& Section 5.1.4) \\
\hline Precipitation of bubbles of & $\begin{array}{l}\text { Calculations of the free energy of Xe bubbles } \\
\text { using rigid ion potential (see Section 5.2.2) }\end{array}$ \\
\hline Resolution of gases & $\begin{array}{l}\text { Calculations of resolution of He and Xe in } \\
\text { cascades and thermal spikes using rigid ion } \\
\text { pair potentials (see Section 5.2.5) }\end{array}$ \\
\hline Radiation induced diffusion & $\begin{array}{l}\text { Investigation using rigid ion pair potentials } \\
\text { (see Section 5.1.8) }\end{array}$ \\
\hline Radiation enhanced diffusion & $\begin{array}{l}\text { Investigation of migration of He in presence of } \\
\text { defects at various stoichiometries (see } \\
\text { Section 5.2.3) }\end{array}$ \\
\hline &
\end{tabular}

Concerning electronic structures calculations, larger and more complex systems need to be modelled and the dynamics of the systems have to be investigated more exhaustively. Then, the influence of the defect charges needs to be studied, as has been started since the end of the project by several authors [82,4446]. The influence of temperature on the parameters calculated should also be determined. The behaviour of the rare gases and the processes near extended defects, as well as the chemical evolution of fuel during burn-up should also be addressed. Moreover, even more sophisticated approximations should be applied to our systems. In particular, the combination of the DFT with the dynamical mean field approximation (DFT + DMFT) [83], which enables a dynamical description of the electronic correlations and permits an even better description of the strong correlations and magnetism in actinide compounds, should in a near future be applicable to defective systems. Then, new functionals developed recently should improve the bonds formed between rare gases and materials. This will enable us to further validate the results presented here.

As for empirical potentials, calculations of even larger systems, especially for the modelling of polycrystalline samples or extended defects, should be envisaged to refine the results presented here. The improvement of the potentials themselves, for instance the development of charge transfer potentials enabling us to take better into account the various charge states exhibited by the actinides in oxide compounds, such as the many-body potentials recently developed by Sattonnay and Tétot [84], Li et al. [85], Cooper et al. [86], would also improve the modelling and will enable us to confirm the results presented in this article.

\subsection{Radiation induced processes}

Table 8 synthesizes the parameters of mesoscale models needed for the description of the radiation induced processes in $\mathrm{UO}_{2}$ which were calculated using atomic scale methods during the F-BRIDGE project. It must be noted that due to the large size of systems that need to be considered and the long times that need to be simulated to model these phenomena, electronic structure methods are not presently applicable. The results shown here were obtained using empirical potentials. 
It can be seen in Table 8 that first calculations have been performed to determine or evaluate all the types of parameters concerning radiation induced processes in $\mathrm{UO}_{2}$ using empirical potentials. These phenomena are, however, numerous and very complex and if interesting results were obtained, the studies presented in this work should mainly be seen as examples of the feasibility of investigating irradiation induced processes at the atomic scale to obtain data necessary for the higher scales. Investigations must be completed to obtain a more exhaustive view of the mechanisms involved. The electronic processes of energy loss in the material must in particular be further addressed. As in the case of the thermal parameters, the application of more sophisticated empirical potentials will also be a source of improvement.

\section{Conclusions}

Despite the specific difficulties involved in the description of fuel under irradiation, the results obtained during the F-BRIDGE project prove that atomic scale modelling methods are now mature enough to investigate fuel materials and enable one to obtain precise data to feed higher scale models and help interpret experiments on nuclear fuel. They have also shown that a multiscale approach aiming at describing the fuel behaviour from the atomic to the mesoscopic scale can contribute significantly in the determination of the underlying phenomena governing the transport properties of defects and rare gases in nuclear fuel.

Due to the complexity of nuclear fuel materials and of the phenomena occurring under irradiation in reactor, however, these results are only one step toward the elucidation of the mechanisms leading to the complete evolution of nuclear fuels under irradiation. Much remains to be done and the results presented in this article on the transport properties of defects and fission gases are being refined and extended.

Progress has already been made in the study of transport properties since the end of the project. The investigation of the influence of the non-stoichiometry on defects [44-46,87] and fission gas behaviour $[82,87,88]$ using DFT + U and the many-body empirical potentials mentioned above [89] have showed the importance of charged defects. Then, the calculation of the diffusion coefficient of Xe calculated using a combination of electronic structure and empirical potential methods have been confronted to the results of atomic diffusion experiments to elucidate the elementary mechanisms involved in gas diffusion [88].

Beyond the transport properties, other phenomena involved in the behaviour of fuel under irradiation are investigated. In particular, thermochemical and thermomechanical properties are the focus of numerous recent studies. Electronic structure calculations of thermal expansion or of the evolution of the bulk modulus as a function of temperature [90], which are important data for macroscale models, have started, as well as the electronic structure evaluation of thermodynamical data, such as specific heat or vibrational entropy [91], which are critical for the determination of phase diagrams and fuel melting temperatures. The $\mathrm{UO}_{2}$ thermal conductivity, which is another key parameter that determines the fuel temperature in the pin, especially at its centre, and therefore impacts strongly all the thermally activated processes, as well as fuel melting in off-normal situations, has also been determined using DFT + U and empirical potentials [92-95]. Then, mechanical properties and their evolution in the presence of irradiation-induced defects have been investigated [96]. The study of the effect of the microstructure, initiated in F-BRIDGE by the investigation of dislocations, grain boundaries and polycrystalline $\mathrm{UO}_{2}$, has also been extended and refined [97,98].

Finally, non-oxide fuel materials have been investigated in and outside F-BRIDGE using electronic structure calculations, in particular carbide [99] and nitride fuels [100-103].
These studies open the way for the investigation of significant phenomena involved in fuel behaviour under irradiation that have not yet been investigated at the atomic scale, in particular.

- The evolution of the chemical composition due to burn-up, and its effect on the fuel characteristics, especially thermal conductivity, melting temperature and mechanical properties.

- Thermodynamic data for fuel and fission product compounds, which are major requirements to derive reliable thermodynamic models and fission product speciation, especially for fuelcladding interaction and off-normal situations.

- Complex microstructures, in particular the high burn-up structure.

- The behaviour under irradiation of more complex fuels, in particular mixed fuels such as MOX and minor actinide bearing fuels.

At the mesoscopic scale, significant effort is needed to develop the models to strengthen further the links between atomic and macroscopic scales and address systematically the problem of transferring information from one scale to another. One specific need is to draw on recent modelling advances to develop and improve elastic, plastic, creep and fracture models. Then, the coupling between mechanics and thermochemistry [104,105] is a promising approach to investigate fuel evolution and fuel cladding interaction.

\section{Acknowledgment}

This research was partly supported by the European Commission through the FP7 F-BRIDGE project (Contract No. 211690).

\section{References}

[1] C. Valot, M. Bertolus, R. Konings, J. Somers, S. de Groot, Nucl. Eng. Des. 241 (2011) 3521.

[2] F-BRIDGE Final report R-012, 2013 <http://cordis.europa.eu/publication/rcn/ 16699_en.html>.

[3] https://inlportal.inl.gov/portal/server.

pt/community/center_for_materials_science_of_nuclear_fuel/655/home.

[4] http://www.ne.anl.gov/NEAMS/.

[5] http://www.casl.gov/.

[6] http://www.nxo.jp.

[7] P. Garcia, G. Carlot, G. Martin, C. Sabathier, M.F. Barthe, T. Belhabib, P. Desgardin, T. Sauvage, E. Gilabert, F. Garrido, Experimental investigations of irradiation induced diffusion of $\mathrm{He}$ and $\mathrm{I}$ in $\mathrm{UO}_{2}$, F-BRIDGE Deliverable D-111, 2013 <http://f-bridge.eu/>.

[8] P. Garcia, G. Martin, C. Sabathier, G. Carlot, A. Michel, P. Martin, B. Dorado, M Freyss, M. Bertolus, R. Skorek, J. Noirot, L. Noirot, O. Kaitasov, S. Maillard, Nucl. Instrum. Methods Res. B 277 (2012) 98.

[9] X.-Y. Liu, D. Andersson, B. Uberuaga, J. Mater. Sci. 47 (2012) 7367.

[10] B. Dorado, M. Freyss, B. Amadon, M. Bertolus, G. Jomard, P. Garcia, J. Phys.: Condens. Matter 25 (2013) 333201.

[11] K. Govers, S. Lemehov, M. Hou, M. Verwerft, J. Nucl. Mater. 366 (2007) 161.

[12] K. Govers, S. Lemehov, M. Hou, M. Verwerft, J. Nucl. Mater. 376 (2008) 66.

[13] R. Devanathan, L. Van Brutzel, A. Chartier, C. Guéneau, A. Mattsson, V. Tikare, T. Bartel, T. Besmann, M. Stan, P. Van Uffelen, Energy Environ. Sci. 3 (2010) 1406.

[14] S. Maillard, R. Skorek, K. Hoang, M. Bertolus, Application of mesoscale approaches to the diffusion processes in $\mathrm{UO}_{2}, \mathrm{~F}-\mathrm{BRIDGE}$ Deliverable D-225 $2012<$ http://f-bridge.eu/>.

[15] R. Skorek, S. Maillard, A. Michel, G. Carlot, E. Gilabert, T. Jourdan, Defect Diffus. Forum 323-325 (2012) 209.

[16] A. Barbu, E. Clouet, Solid State Phenom. 129 (2007) 51.

[17] L. Noirot, Nucl. Eng. Des. 241 (2011) 2099.

[18] G. Martin, P. Garcia, C. Sabathier, L. Van Brutzel, B. Dorado, F. Garrido, S. Maillard, Phys. Lett. A 374 (2010) 3038.

[19] E.W. Elcock, C.W. McCombie, Phys. Rev. 109 (1958) 605.

[20] E.W. Elcock, Proc. Phys. Soc. 73 (1959) 250.

[21] A.B. Bortz, M.H. Kalos, J.L. Lebowitz, J. Comput. Phys. 17 (1975) 10.

[22] D. Gillespie, J. Comput. Phys. 22 (1976) 403.

[23] D. Gillespie, J. Phys. Chem. 81 (1977) 2340.

[24] A.F. Voter, Introduction to the kinetic Monte Carlo method, in: K.E. Sickafus, E.A. Kotomin (Eds.), Radiation Effects in Solids, Springer, NATO Publishing Unit, Dordrecht, The Netherlands, 2005.

[25] G. Martin, Phys. Rev. B 41 (1990) 2279. 
[26] G. Martin, F. Soisson, Kinetic Monte Carlo method to model diffusion controlled phase transformations in the solid state, in: S. Yip (Ed.), Handbook of Materials Modeling, Springer, Berlin, 2005.

[27] M. Wen, A. Takahashi, N.M. Ghoniem, J. Nucl. Mater. 392 (2009) 386.

[28] L. Malerba, C.S. Becquart, C. Domain, J. Nucl. Mater. 360 (2007) 159.

[29] C.S. Deo, D.J. Srolovitz, W. Cai, V.V. Bulatov, J. Mech. Phys. Solids 53 (2005) 1223.

[30] J. Dalla Torre, J.-L. Bocquet, N.V. Doan, E. Adam, A. Barbu, Philos. Mag. 85 (2005) 549.

[31] D.A. Andersson, T. Watanabe, C. Deo, B.P. Uberuaga, Phys. Rev. B 80 (R) (2009) 060101.

[32] J.F. Marin, P. Contamin, J. Nucl. Mater. 30 (1969) 16.

[33] P. Contamin, J. Bacmann, J. Marin, J. Nucl. Mater. 42 (1972) 54.

[34] R.M. Martin, Electronic Structure: Basic Theory and Practical Methods, Cambridge University Press, Cambridge, 2004.

[35] U. Burkert, N.L. Allinger, Molecular Mechanics, ACS Monographs, American Chemical Society, Washington, DC, 1982.

[36] M. Bertolus, M. Freyss, G. Martin, B. Dorado, L. Van Brutzel, A. Chartier, R. Grimes, D. Parfitt, C. Bishop, M. Krack, F. Devynck, K. Govers, Development and assessment of atomistic modelling methods for the investigation of fuel materials under operational conditions, F-BRIDGE Deliverable D-221, 2010 $<$ http://f-bridge.eu/>.

[37] M. Bertolus, M. Major, V. Brenner, Phys. Chem. Chem. Phys. 14 (2012) 553.

[38] C.L. Bishop, S.T. Murphy, M.J.D. Rushton, R.W.M. Grimes, Nucl. Inst. Methods B 274 (2012) 195.

[39] B. Dorado, B. Amadon, M. Freyss, M. Bertolus, Phys. Rev. B 79 (2009) 235125.

[40] B. Dorado, B. Amadon, G. Jomard, M. Freyss, M. Bertolus, Phys. Rev. B 84 (2011) 096101.

[41] B. Dorado, M. Freyss, G. Jomard, M. Bertolus, Phys. Rev. B 82 (2010) 035114.

[42] A. Kotani, T. Yamazaki, Prog. Theor. Phys. 108 (Suppl.) (1992) 117. 131.

[43] B. Dorado, Etude des propriétés de transport atomique dans le dioxyde d'uranium par le calcul de structure électronique : influence des fortes corrélations, Ph.D. thesis, Université de la Méditerranée Aix-Marseille II, 2010 $<$ http://www.theses.fr/150328060>.

[44] P. Nerikar, T. Watanabe, J.S. Tulenko, S.R. Phillpot, S.B. Sinnott, J. Nucl. Mater. 384 (2009) 61

[45] J.-P. Crocombette, Phys. Rev. B 85 (2012) 144101.

[46] E. Vathonne, J. Wiktor, M. Freyss, G. Jomard, M. Bertolus, J. Phys.: Condens. Matter 26 (2014) 325501.

[47] B. Dorado, J. Durinck, M. Freyss, M. Bertolus, P. Garcia, J. Nucl. Mater. 400 (2010) 103.

[48] B. Dorado, P. Garcia, G. Carlot, C. Davoisne, M. Fraczkiewicz, B. Pasquet, M. Freyss, C. Valot, G. Baldinozzi, D. Simeone, M. Bertolus, Phys. Rev. B 83 (2011) 035126.

[49] B. Dorado, D.A. Andersson, C.R. Stanek, M. Bertolus, B.P. Uberuaga, G. Martin, M. Freyss, P. Garcia, Phys. Rev. B 86 (2012) 035110.

[50] G. Henkelman, H. Jonsson, J. Chem. Phys. 113 (2000) 9978.

[51] A. Laio, M. Parrinello, Proc. Natl. Acad. Sci. 99 (2002) 12562.

[52] M. Iannuzzi, A. Laio, M. Parrinello, Phys. Rev. Lett. 90 (2003) 238302.

[53] CP2K developers group 2000-2012 <http://www.cp2k.org>.

[54] N.-D. Morelon, D. Ghaleb, J.-M. Delhaye, L. Van Brutzel, Philos. Mag. 83 (2003) 1533.

[55] C. Meis, A. Chartier, J. Nucl. Mater. 341 (2005) 25.

[56] E. Vincent-Aublant, L. Van Brutzel, J.M. Delaye, J. Nucl. Mater. 392 (2009) 114

[57] R. Grimes, D. Parfitt, S. Murphy, L. Van Brutzel, K. Govers, Empirical potential investigation of transport properties in polycrystalline $\mathrm{UO}_{2}$ and in the presence of extended defects, F-BRIDGE Deliverable D-223, $2012<$ http://fbridge.eu/>.

[58] L. Van Brutzel, E. Vincent-Aublant, J. Nucl. Mater. 377 (2008) 522.

[59] A.C.S. Sabioni, W.B. Ferraz, F. Millot, J. Nucl. Mater. 257 (1998) 180.

[60] A.C.S. Sabioni, W.B. Ferraz, F. Millot, J. Nucl. Mater. 278 (2000) 364.

[61] F. Devynck, M. Iannuzzi, M. Krack, Phys. Rev. B 85 (2012) 184103.

[62] D.C. Parfitt, C.L. Bishop, M.R. Wenman, R.W. Grimes, J. Phys.: Condens. Matter 22 (2010) 175004

[63] R.J. Keller, T.E. Mitchell, A.H. Heuer, Acta Metall. 36 (1988) 1073.

[64] C.L. Bishop, R.W. Grimes, D.C. Parfitt, Nucl. Inst. Methods B 268 (2010) 2915.

[65] L. Van Brutzel, E. Aublant-Vincent, Nucl. Instrum. Methods B 267 (2009) 3013.
[66] G. Martin, S. Maillard, L. Van Brutzel, P. Garcia, B. Dorado, C. Valot, J. Nucl. Mater. 385 (2009) 351.

[67] A.E. Thompson, C. Wolverton, Phys. Rev. B 84 (2011) 134111.

[68] G. Brillant, F. Gupta, A. Pasturel, J. Nucl. Mater. 170 (2011).

[69] S. Murphy, A. Chartier, L. Van Brutzel, J.P. Crocombette, Phys. Rev. B 85 (2012) 144102.

[70] A. Chartier, L. Van Brutzel, M. Freyss, Phys. Rev. B 81 (2010) 174111.

[71] E. Yakub, C. Ronchi, D. Staicu, J. Nucl. Mater. 389 (2009) 119.

[72] K. Govers, S.E. Lemehov, M. Hou, M. Verwerft, J. Nucl. Mater 395 (2009) 131.

[73] C. Basak, A. Sengupta, H. Kamath, J. Alloys Compd. 360 (2003) 210.

[74] K. Govers, S.E. Lemehov, M. Verwerft, J. Nucl. Mater. 405 (2010) 252.

[75] K. Govers, S.E. Lemehov, M. Verwerft, Defect Diffus. Forum 323-325 (2012) 215.

[76] P.V. Nerikar, D.C. Parfitt, L.A. Casillas Trujillo, A.A. Andersson, C. Unal, S.B. Sinnott, R.W. Grimes, B.P. Uberuaga, C.R. Stanek, Phys. Rev. B 84 (2011) 174105.

[77] R.S. Nelson, J. Nucl. Mater. 25 (1968) 227.

[78] R.M. Cornell, J.A. Turnbull, J. Nucl. Mater. 41 (1971) 87.

[79] D.C. Parfitt, R.W. Grimes, J. Nucl. Mater. 381 (2008) 216.

[80] D.C. Parfitt, R.W. Grimes, J. Nucl. Mater. 392 (2009) 28.

[81] K. Govers, C.L. Bishop, D.C. Parfitt, S.E. Lemehov, M. Verwerft, R.W. Grimes, J. Nucl. Mater. 420 (2012) 282.

[82] D. Andersson, B. Uberuaga, P. Nerikar, C. Unal, C. Stanek, Phys. Rev. B 84 (2011) 054105.

[83] A. Georges, G. Kotliar, W. Krauth, M.J. Rozenberg, Rev. Mod. Phys. 68 (1996) 13.

[84] G. Sattonnay, R. Tétot, J. Phys.: Condens. Matter 25 (2013) 125403.

[85] Y. Li, T. Liang, S.B. Sinnott, S.R. Phillpot, J. Phys.: Condens. Matter. 25 (2013) 505401.

[86] M.W.D. Cooper, M.J.D. Rushton, R.W. Grimes, J. Phys.: Condens. Matter. 26 (2014) 105401.

[87] E. Vathonne, Étude par calcul de structure électronique des dégâts d'irradiation dans le combustible nucléaire $\mathrm{UO}_{2}$ : comportement des défauts ponctuels et gaz de fission, Ph.D. Thesis, Aix-Marseille Université, 2014.

[88] D. Andersson, P. Garcia, X.-Y. Liu, G. Pastore, M. Tonks, P. Millett, B. Dorado, D. Gaston, D. Andrs, R. Williamson, R. Martineau, B. Uberuaga, C. Stanek, J. Nucl. Mater. 451 (2014) 225.

[89] M.W.D. Cooper, S.C. Middleburgh, R.W. Grimes, Solid State Ionics 266 (2014) 68.

[90] B.-T. Wang, P. Zhang, R. Lizárraga, I. Di Marco, O. Eriksson, Phys. Rev. B 88 (2013) 104107.

[91] M. Sanati, R. Albers, T. Lookman, A. Saxena, Phys. Rev. B 84 (2011) 014116.

[92] S. Nichenko, D. Staicu, J. Nucl. Mater. 433 (2013) 297.

[93] M.J. Qin, M.W.D. Cooper, E.Y. Kuo, M.J.D. Rushton, R.W. Grimes, G.R. Lumpkin, S.C. Middleburgh, J. Phys.: Condens. Matter 26 (2014) 495401.

[94] J.W.L. Pang, W.J.L. Buyers, A. Chernatynskiy, M.D. Lumsden, B.C. Larson, S.R. Phillpot, Phys. Rev. Lett. 110 (2013) 157401.

[95] B. Deng, A. Chernatynskiy, P. Shukla, S.B. Sinnott, S.R. Phillpot, J. Nucl. Mater. 434 (2013) 203.

[96] P. Fossati, Contribution à l'étude des propriétés mécaniques du combustible nucléaire : Modélisation atomistique de la déformation du dioxyde d'uranium, Ph.D thesis, Ecole Centrale Paris, $2012<$ http://www.theses.fr/ 2012ECAP0045>.

[97] T.-W. Chiang, A. Chernatynskiy, B. Deng, S.B. Sinnott, S.R. Phillpot, J. Nucl. Mater. 448 (2014) 53.

[98] P. Fossati, L. Van Brutzel, B. Devincre, J. Nucl. Mater. 443 (2013) 359.

[99] M. Freyss, Phys. Rev. B 81 (2010) 014101.

[100] E.A. Kotomin, R.W. Grimes, Y. Mastrikov, N.J. Ashley, J. Phys.: Condens. Matter 19 (2007) 106208.

[101] D. Gryaznov, E. Heifets, E.A. Kotomin, Phys. Chem. Chem. Phys. 14 (2012) 4482.

[102] M. Klipfel, P. Van Uffelen, J. Nucl. Mater. 422 (2012) 137.

[103] M. Pukari, P. Olsson, N. Sandberg, J. Nucl. Mater. 438 (2013) 7.

[104] B. Baurens, J. Sercombe, C. Riglet-Martial, L. Desgranges, L. Trottignon, P. Maugis, J. Nucl. Mater. 452 (2014) 578.

[105] M. Piro, J. Banfield, K. Clarno, S. Simunovic, T. Besmann, B. Lewis, W. Thompson, J. Nucl. Mater. 441 (2013) 240. 